\title{
Les horaires et l'organisation du temps de travail
}

\begin{abstract}
Alain Chenu*
La récente enquête Emploi du temps réalisée par l'Insee - avant que les lois sur les 35 heures n'entrent en application - fait appel à un mode de questionnement inédit décrivant quart d'heure par quart d'heure, au long d'une semaine entière, les horaires du travail professionnel. D'une très grande diversité, ils peuvent être répartis, pour les actifs occupés à temps plein, en six grands types professionnels.
\end{abstract}

Les entrepreneurs ont la liberté d'organiser leur temps à leur convenance. Leurs horaires de travail sont lourds et débordent sur les soirées et les fins de semaine. Les enseignants ont des horaires assez légers, répartis en de nombreux épisodes plutôt brefs, se situant pour partie à domicile, à des heures tardives et durant le week-end. Les experts, managers et gestionnaires figurent en position intermédiaire entre les entrepreneurs et les salariés d'exécution : ils organisent leur temps de travail avec une assez grande liberté, se déclarent souvent «débordés » et leur vie professionnelle empiète sur leur temps familial et domestique. Mais ils travaillent moins que les autres salariés la nuit ou durant le week-end. Les professionnels des services aux personnes (infirmières, employés de commerce, femmes de ménage, etc.) ont des horaires fréquemment irréguliers. Ils travaillent beaucoup en fin d'après-midi ou en début de soirée, quand les autres salariés ont fini leur journée. Les agents des grandes organisations bureaucratiques sont les plus nombreux à pratiquer une semaine standard de cinq jours consécutifs avec horaire diurne régulier. La séparation entre vie professionnelle et autres temps sociaux est pour eux particulièrement nette. Un contrôle hiérarchique assez contraignant s'exerce sur leur temps de travail. Enfin, les ouvriers de l'industrie, les policiers et militaires se distinguent du type précédent par la fréquence assez élevée des horaires de nuit.

À durée du travail identique, bénéficier d'horaires standard régulièrement répartis sur cinq journées favorise l'expression du sentiment de disposer de temps, et, inversement, être responsable de l'organisation de son temps de travail renforce le sentiment d'être débordé. 
$\mathbf{L}$ a durée et l'organisation du temps de travail dépendent d'un très grand nombre de facteurs : principalement des caractéristiques collectives propres du secteur d'activité et de la profession exercée. Les horaires sont habituellement plus lourds, par exemple, dans la boulangerie que dans la grande industrie, dans le commerce que dans l'enseignement, les horaires réguliers plus fréquents dans les bureaux que dans les hypermarchés, etc. Elles varient aussi en fonction de préférences et de disponibilités individuelles ou familiales.

L'enquête française sur les emplois du temps, réalisée de février 1998 à février 1999 auprès de 15441 personnes de 15 ans ou plus (Dumontier et Pan Ke Shon, 1999 ; Dumontier et Pan Ke Shon, 2000), fait appel à un type de questionnaire nouveau qui décrit les horaires de travail au long d'une semaine entière (cf. encadré 1). Cette source statistique permet d'enrichir la connaissance de la durée du travail, et surtout celle des formes d'organisation du temps de travail. Pour l'essentiel, cette enquête s'est déroulée avant que ne commencent à se faire sentir les effets du passage à la semaine de 35 heures (lois du 13 juin 1998 et du 19 janvier 2000) ; mais les informations qu'elle fournit sont susceptibles d'éclairer des débats qui se sont développés dans le contexte de la mise en œuvre de politiques de «RTT» (réduction du temps de travail), et qui ont porté non seulement sur la durée du travail, mais aussi sur l'organisation du temps de travail. L'enquête de 1998-1999 permet de comparer, à durée du travail donnée, les caractéristiques des personnes effectuant différents types d'horaires, et d'étudier leur perception de cette durée et de l'organisation du travail qui lui est associée.

On se limite ici à l'étude de l'organisation des journées et des semaines normales de travail des actifs occupés à temps plein, en laissant de côté l'analyse des situations à temps partiel et celle des périodes de congés (une « semaine normale de travail » est ici une semaine déclarée comme telle par la personne qui remplit le « semainier »; elle s'oppose aux semaines de congés, chômées, et autres - travail et congés, etc.) (1). Les personnes qui effectuent de longues semaines de travail prennent généralement moins de congés que celles dont les semaines sont relativement légères (Boisard et Fermanian, 1999). L'enquête Emploi du temps permet seulement de constater que les semaines longues vont de pair avec de fortes proportions de semaines normales de travail - et avec de faibles proportions de semaines comportant des congés. En revanche, cette enquête permet d'analyser la répartition des types de journée et de semaine, ainsi que le rapport au temps de travail, plus subjectif, en fonction des principales caractéristiques des enquêtés (sexe, âge, diplôme, statut d'emploi et catégorie socioprofessionnelle). Elle permet également de cerner le rôle des contraintes techniques et des conventions sociales qui confèrent leur caractère spécifique aux horaires de travail propres à tel secteur ou à telle profession. Elle permet enfin de caractériser les déterminants du vécu subjectif du temps de travail, en identifiant les types d'horaires et les formes de gestion du temps de travail qui, la durée du travail étant contrôlée, favorisent ou limitent l'expression du sentiment d'être débordé.

\section{L'organisation des horaires de travail}

$\mathbf{L}$ a multiplicité des horaires est grande selon la profession exercée. Ainsi, dans le cas de certains entrepreneurs individuels, le travail empiète sur le repos hebdomadaire du week-end au point que ces personnes déclarent travailler six ou sept jours sur sept. D'autres professions (infirmières, policiers et militaires) sont tenues au travail de nuit, qui est sans doute plus facile pour des jeunes que pour des personnes plus âgées. Les enseignants travaillent tard en soirée pour préparer leurs cours. Le type d'horaires pratiqués (quotidien ou hebdomadaire) dépend des caractéristiques individuelles (âge, sexe, diplôme, statut professionnel).

\section{Les jeunes et les cadres sont peu matinaux}

Les journées sont découpées en sept tranches horaires. La nuit (de 23 h à 5 h) est encadrée par deux tranches longues chacune de quatre heures, la soirée et le petit matin. Les dix heures diurnes restantes sont réparties en quatre plages de deux heures trente - matin de 9 h à 11 h 30 , midi de $11 \mathrm{~h} 30$ à $14 \mathrm{~h}$, début d'après-midi de $14 \mathrm{~h}$ à $16 \mathrm{~h}$ 30, fin d'après-midi de 16 h 30 à $19 \mathrm{~h}$.

1. La très grande diversité des formules de travail à temps partiel requerrait à elle seule une étude spécifique. L'information sur la durée des congés est centrale dans toute étude d'ensemble des formes d'organisation du temps de travail. Elle est malheureusement de mauvaise qualité dans l'enquête Emploi du temps de 1998-1999, du fait de fréquentes confusions entre les réponses en nombre de jours et celles en nombre de semaines. 
Encadré 1

\section{LE SEMAINIER}

Une des limites de la plupart des enquêtes sur les emplois du temps tient au fait que chaque personne remplit un carnet d'activités couvrant une seule journée - tantôt de semaine, tantôt de week-end - et que le coût de ce questionnement limite la taille des échantillons; par suite les informations sur les durées et le rythme des activités observées sont entachées de fortes fluctuations aléatoires. Un questionnement sur une semaine entière permet de cerner les horaires de travail avec plus de précision statistique puisqu'il porte sur sept fois plus de journées.

L'idée d'inclure un "semainier " dans des enquêtes sur les emplois du temps a pris naissance dans le cadre d'une réunion de l'unité Emploi de la DG V de la Commission des Communautés Européennes en 1994. Les experts préparaient les enquêtes sur les emplois du temps qui devaient se dérouler dans la plupart des pays de la communauté à partir de 1999. L'amélioration de la connaissance des horaires effectifs de travail apparaissant comme une priorité, les experts - notamment liris Niemi, responsable du projet sur les enquêtes Emploi du temps à Eurostat, et Klas Rydenstam, de Statistic Sweden - ont décidé de mettre au point un questionnement au long d'une semaine. Un test concluant ayant été effectué en Suède et en Italie en 1995, un semainier a été inclus dans l'enquête pilote réalisée en 1996-1997. S'ils étaient en emploi, les répondants devaient décrire de manière rétrospective, quart d'heure par quart d'heure, la semaine se terminant au jour décrit au carnet d'activités. La comparaison, pour 1604 personnes, entre durée du travail observée au carnet quotidien et durée pour la même journée observée au semainier a mis en évidence des divergences non négligeables, de sorte qu'Eurostat a décidé de ne pas recommander l'inclusion de ce semainier dans les enquêtes nationales à venir. Cependant plusieurs pays - notamment la France et la Finlande - ont jugé ce dispositif prometteur et l'ont inclus dans leur enquête Emploi du temps.

Dans le cas français, au cours d'une première visite, si au moins une personne en emploi vivait dans le ménage, l'enquêteur présentait le semainier et donnait les consignes correspondantes: les temps de repas, les pauses, les déplacements domicile-travail ne doivent pas être inclus dans le temps de travail, l'emploi indépendant, l'aide apportée à un membre de la famille, le travail rapporté à la maison doivent être inclus. Les personnes avaient à remplir le semainier jour après jour et à le renvoyer à l'Insee, sous une enveloppe pré-affranchie. Cette procédure préservait mieux des défaillances de mémoire que celle testée par Eurostat, mais certaines personnes ont omis de renvoyer le formulaire, même après un rappel téléphonique. Au total, $79,2 \%$ des personnes en emploi éligibles à l'enquête et $86,6 \%$ de celles qui ont rempli un carnet quotidien d'activités exploitable ont renvoyé un semainier. Quatre fois sur cinq ce semainier concerne une semaine normale de travail, dans $6 \%$ des cas une semaine de congés (non compris les congés passés ailleurs qu'à la résidence principale) et pour le reste, $14 \%$, une semaine autre, c'est-à-dire mixte.

La durée du travail d'après le carnet d'activités est un peu inférieure à celle observée pour la même journée sur le semainier. Parmi les 2428 actifs occupés à temps plein ayant décrit une même journée de travail au carnet d'une part, et au semainier (dans le cadre d'une semaine de travail normale) d'autre part, la durée moyenne est de 8,3 heures au carnet et 8,7 heures au semainier.
Pour 6463 personnes en emploi interrogées dans le cadre de l'enquête Emploi du temps, on dispose à la fois des informations issues du carnet d'activités, de celles du semainier, de l'horaire hebdomadaire estimé, et des renseignements socio-démographiques usuels (âge, diplôme, profession).

Ce sous-échantillon - dont les semainiers décrivent donc environ 45000 journées - fait l'objet d'une pondération spécifique. Les effectifs selon l'âge et la profession observés aux enquêtes Emploi de mars 1998 et janvier 1999 sont pris comme référence. Pour les ensembles de profession de petite taille (CS 44 (clergé), 23 (chefs d'entreprise), 35 (professions de l'information, des arts et des spectacles), 34 (professeurs, professions scientifiques), 31 (professions libérales), 33 (cadres administratifs de la Fonction publique), 21 (artisans),64 (chauffeurs), 48 (contremaîtres)), l'âge n'entre pas dans le redressement. Pour les autres, trois groupes d'âge sont distingués (moins de 25 ans, 25 à 54 ans, 55 ans et plus). L'échantillon non redressé sous-représenterait les plus jeunes et les moins qualifiés des actifs en emploi. Les coefficients de pondération s'étagent de 2467 à 6843 , avec une moyenne de 3508 .

Le graphique de l'encadré 2 donne quatre exemples de semainiers présentant des caractéristiques contrastées : horaire lourd d'un agriculteur, semaine standard d'un ingénieur, horaire irrégulier et fragmenté d'un professeur, travail de nuit d'une infirmière s'organisant en trois longs épisodes.

Un rapprochement avec la moyenne des enquêtes Emploi de mars 1998 et de janvier 1999 montre que, dans le champ des actifs à temps plein décrivant une semaine normale de travail (1), les horaires décrits au semainier excèdent de $3,3 \%$ ceux estimés à l'enquête Emploi. Les professions qui contribuent à cet écart sont principalement les enseignants (à l'enquête Emploi les professeurs sous-estiment leur temps de préparation de cours et de corrections; leurs horaires au semainier sont de $18 \%$ supérieurs aux estimations fournies à l'enquête Emploi ; chez les instituteurs et professeurs des écoles l'écart est de $+10 \%$ ), les agriculteurs (+ $14 \%$ ), les personnels de service à l'exception des assistantes maternelles (+ $9 \%$ ), les techniciens et les professions intermédiaires des entreprises $(+7$ et $+8 \%$ ), les chauffeurs $(+7 \%)$; ils semblent inclure dans le temps de travail décrit au semainier des phases d'attente qui ne sont que partiellement prises en compte dans l'horaire estimé à l'enquête Emploi, plus proche du temps rémunéré). Inversement les horaires des assistantes maternelles sont plus légers de $8 \%$ au semainier qu'à l'enquête Emploi - du temps de présence responsable, inclus dans la durée estimée à l'enquête Emploi, est exclu du temps de travail décrit au semainier. Dans l'ensemble les estimations de l'enquête Emploi se rapprochent d'une durée contractuelle ou d'une durée servant au calcul de la rémunération. Mais des variations saisonnières et des fluctuations aléatoires contribuent aussi aux écarts entre les deux sources.

1. Les valeurs observées à l'enquête Emploi sont les estimations de la durée du travail effective au cours de la semaine de référence, dans le champ des personnes ayant travaillé comme d'habitude. 
$80 \%$ du temps de travail professionnel se concentrent de $9 \mathrm{~h}$ à $19 \mathrm{~h}$. Ce taux de concentration diurne atteint $84 \%$ chez les femmes et $77 \%$ seulement chez les hommes (cf. tableau 1).

Les moins de 30 ans effectuent près de $3 \%$ de leurs heures dans la tranche nocturne entre $23 \mathrm{~h}$ et $5 \mathrm{~h}$, les 50 ans et plus $1,5 \%$ seulement. Les plus jeunes sont légèrement moins matinaux que les plus âgés : $35 \%$ du temps de travail des moins de 30 ans est accompli de 5 h à 11 h 30, contre $37 \%$ de celui des 50 ans et plus.

Plus on est diplômé, plus on commence à travailler tard le matin, et plus on finit tard le soir. C'est particulièrement apparent pour la tranche du petit matin, de $5 \mathrm{~h}$ à $9 \mathrm{~h}$ : les moins diplômés (sans diplôme et titulaires du certificat d'études primaires, auxquels on assimile les répondants qui ne renseignent pas leur niveau de diplôme) y effectuent $15 \%$ du total de leurs heures, les titulaires d'un diplôme supérieur au baccalauréat, $7 \%$ seulement. De la même manière, les cadres et assimilés sont peu matinaux, à l'opposé des employés et ouvriers non qualifiés (cf. graphique I).

Le travail de nuit est plus fréquent dans la Fonction publique et dans les grands et moyens établissements que chez les non salariés et les salariés des petits établissements; les non salariés fournissent beaucoup de travail en fin de journée - mais très peu de travail de nuit.

\section{Six types de semaines de travail}

On analyse le lien entre horaires et caractéristiques individuels au regard d'une typologie $a$ priori des horaires hebdomadaires. Celle-ci s'appuie sur les informations disponibles dans le semainier. Elle comporte six types de semaines de travail: semaine avec travail de nuit, semaine comportant au moins six jours de travail, semaine standard, semaine de cinq jours (sauf semaine standard), semaine de quatre jours de travail, autres semaines (cf. encadré 2).

Tableau 1

Répartition du temps de travail par tranches horaires

\begin{tabular}{|c|c|c|c|c|c|c|c|c|c|c|c|}
\hline & \multicolumn{2}{|c|}{$\begin{array}{l}\text { Semaines } \\
\text { normales }\end{array}$} & \multicolumn{2}{|c|}{$\begin{array}{c}\text { Horaire } \\
\text { hebdomadaire }\end{array}$} & \multicolumn{7}{|c|}{ Tranche horaire (en \%) } \\
\hline & \begin{tabular}{|c} 
Part \\
des \\
semai- \\
niers \\
$($ en \%) \\
$(1)$
\end{tabular} & $\begin{array}{l}\text { Struc- } \\
\text { tures } \\
\text { (en \%) }\end{array}$ & $\begin{array}{c}\text { Moyen- } \\
\text { ne } \\
(\mathrm{h} / \mathrm{sem} .)\end{array}$ & $\begin{array}{c}\text { Écart- } \\
\text { type }\end{array}$ & $\begin{array}{l}\text { Nuit } \\
(23 \mathrm{~h}- \\
5 \mathrm{~h})\end{array}$ & $\begin{array}{c}\text { Petit } \\
\text { matin } \\
(5 \mathrm{~h}- \\
9 \mathrm{~h})\end{array}$ & $\begin{array}{c}\text { Matin } \\
(9 \mathrm{~h}- \\
11 \mathrm{~h} \mathrm{30})\end{array}$ & $\begin{array}{c}\text { Midi } \\
(11 \mathrm{~h} 30- \\
14 \mathrm{~h})\end{array}$ & $\begin{array}{c}\text { Début } \\
\text { d'après- } \\
\text { midi } \\
\text { d'après- } \\
\text { midi }\end{array}$ & $\begin{array}{c}\text { Fin } \\
\text { d'après- } \\
\text { midi } \\
16 \mathrm{~h} 30- \\
19 \mathrm{~h})\end{array}$ & $\begin{array}{l}\text { Soirée } \\
(19 \text { h- } \\
23 \text { h) }\end{array}$ \\
\hline $\begin{array}{l}\text { Homme } \\
\text { Femme }\end{array}$ & $\begin{array}{l}81,4 \\
78,5\end{array}$ & $\begin{array}{l}64,4 \\
35,7\end{array}$ & $\begin{array}{l}45,2 \\
41,2\end{array}$ & $\begin{array}{l}13,0 \\
10,7\end{array}$ & $\begin{array}{l}2,6 \\
1,2 \\
\end{array}$ & $\begin{array}{r}12,6 \\
9,2 \\
\end{array}$ & $\begin{array}{l}24,4 \\
25,8\end{array}$ & $\begin{array}{l}15,5 \\
17,7\end{array}$ & $\begin{array}{l}22,0 \\
23,6\end{array}$ & $\begin{array}{l}16,0 \\
16,6\end{array}$ & $\begin{array}{l}6,9 \\
5,9\end{array}$ \\
\hline $\begin{array}{l}\text { Moins de } 30 \text { ans } \\
30 \text { à } 49 \text { ans } \\
50 \text { ans et plus }\end{array}$ & $\begin{array}{l}78,9 \\
80,7 \\
80,5 \\
\end{array}$ & $\begin{array}{l}19,7 \\
59,6 \\
20,6\end{array}$ & $\begin{array}{l}42,0 \\
43,7 \\
45,6\end{array}$ & $\begin{array}{l}10,9 \\
12,2 \\
13,6 \\
\end{array}$ & $\begin{array}{l}2,8 \\
2,1 \\
1,7 \\
\end{array}$ & $\begin{array}{l}11,3 \\
11,3 \\
11,9 \\
\end{array}$ & $\begin{array}{l}24,3 \\
25,1 \\
24,8 \\
\end{array}$ & $\begin{array}{l}16,4 \\
16,4 \\
15,7 \\
\end{array}$ & $\begin{array}{l}22,5 \\
22,6 \\
22,1 \\
\end{array}$ & $\begin{array}{l}15,9 \\
16,1 \\
16,8 \\
\end{array}$ & $\begin{array}{l}6,9 \\
6,3 \\
7,0 \\
\end{array}$ \\
\hline $\begin{array}{l}\text { Sans diplôme, CEP, sans réponse } \\
\text { CAP, BEP } \\
\text { Baccalauréat au plus } \\
\text { Diplôme supérieur au baccalauréat }\end{array}$ & $\begin{array}{l}83,5 \\
80,5 \\
79,2 \\
78,2\end{array}$ & $\begin{array}{l}20,2 \\
40,6 \\
13,5 \\
25,7\end{array}$ & $\begin{array}{l}43,8 \\
43,7 \\
43,5 \\
43,9\end{array}$ & $\begin{array}{l}13,6 \\
12,1 \\
11,8 \\
12,1\end{array}$ & $\begin{array}{l}2,9 \\
2,3 \\
2,2 \\
1,3\end{array}$ & $\begin{array}{r}15,0 \\
13,3 \\
9,7 \\
6,6\end{array}$ & $\begin{array}{l}24,5 \\
24,8 \\
25,1 \\
25,2\end{array}$ & $\begin{array}{l}15,9 \\
16,0 \\
16,3 \\
16,9\end{array}$ & $\begin{array}{l}20,9 \\
22,1 \\
23,2 \\
24,0\end{array}$ & $\begin{array}{l}14,0 \\
15,4 \\
17,1 \\
19,0\end{array}$ & $\begin{array}{l}6,8 \\
6,1 \\
6,5 \\
7,0\end{array}$ \\
\hline $\begin{array}{l}\text { Salarié de la Fonction publique } \\
\text { Salarié d'un grand étab. } \\
\text { (plus de } 500 \text { sal.) } \\
\text { Salarié d'un étab. de } 10 \\
\text { à } 499 \text { salariés } \\
\text { Salarié d'un petit étab. } \\
\text { (moins de } 10 \text { sal.) } \\
\text { Non salarié }\end{array}$ & $\begin{array}{l}70,6 \\
81,0 \\
82,0 \\
84,1 \\
87,7\end{array}$ & $\begin{array}{l}20,0 \\
13,2 \\
37,0 \\
17,6 \\
12,2 \\
\end{array}$ & $\begin{array}{l}39,6 \\
40,8 \\
42,1 \\
45,4 \\
56,7\end{array}$ & $\begin{array}{r}10,6 \\
8,5 \\
9,2 \\
12,1 \\
17,2 \\
\end{array}$ & $\begin{array}{l}2,5 \\
2,9 \\
2,4 \\
1,6 \\
1,1 \\
\end{array}$ & $\begin{array}{r}10,6 \\
11,6 \\
12,8 \\
9,5 \\
11,6 \\
\end{array}$ & $\begin{array}{l}26,4 \\
24,2 \\
25,0 \\
25,3 \\
23,1 \\
\end{array}$ & $\begin{array}{l}17,3 \\
17,3 \\
16,3 \\
16,0 \\
14,6 \\
\end{array}$ & $\begin{array}{l}23,4 \\
23,4 \\
22,9 \\
23,2 \\
19,1\end{array}$ & $\begin{array}{r}14,0 \\
14,7 \\
15,2 \\
\\
18,4 \\
19,8 \\
\end{array}$ & $\begin{array}{r}5,8 \\
6,0 \\
5,5 \\
6,1 \\
10,9\end{array}$ \\
\hline $\begin{array}{l}\text { Cadres et assimilés } \\
\text { Professions intermédiaires } \\
\text { Employés et ouvriers qualifiés } \\
\text { Employés et ouvriers non qualifiés }\end{array}$ & $\begin{array}{l}79,2 \\
74,4 \\
80,8 \\
81,5\end{array}$ & $\begin{array}{l}13,2 \\
20,4 \\
34,9 \\
19,1\end{array}$ & $\begin{array}{l}45,1 \\
41,7 \\
40,4 \\
41,6\end{array}$ & $\begin{array}{r}11,6 \\
9,8 \\
8,5 \\
10,2 \\
\end{array}$ & $\begin{array}{l}0,7 \\
1,9 \\
3,2 \\
2,4\end{array}$ & \begin{tabular}{|r|}
6,8 \\
9,2 \\
13,2 \\
14,3 \\
\end{tabular} & $\begin{array}{l}25,1 \\
25,8 \\
25,8 \\
24,2 \\
\end{array}$ & $\begin{array}{l}16,7 \\
16,8 \\
16,4 \\
16,9 \\
\end{array}$ & $\begin{array}{l}24,0 \\
24,4 \\
23,3 \\
21,4 \\
\end{array}$ & $\begin{array}{l}19,9 \\
16,5 \\
13,4 \\
14,5 \\
\end{array}$ & $\begin{array}{l}6,8 \\
5,4 \\
4,8 \\
6,3 \\
\end{array}$ \\
\hline Ensemble & 80,3 & 100,0 & 43,8 & 12,3 & 2,1 & 11,5 & 24,9 & 16,3 & 22,5 & 16,2 & 6,5 \\
\hline
\end{tabular}

1. 5283 répondants travaillant à temps plein ont fourni un semainier. 4224 semainiers décrivent une semaine de travail normale. La collecte de l'enquête Emploi du temps s'étale sur une année, mais est interrompue durant deux semaines fin décembre-début janvier, et deux semaines début août.

Lecture : 2,1 \% en moyenne des actifs occupés à temps plein travaillent de nuit (pour la définition du travail de nuit, voir encadré 2). Champ : actifs occupés à temps plein et décrivant une semaine normale de travail.

Source : enquête Emploi du temps, 1998-1999, Insee. 
Forme a priori courante d'organisation des horaires, la semaine standard est en fait relativement minoritaire (cf. tableau 2): elle ne concerne que $32 \%$ des actifs occupés à temps plein $(27 \%$ si on exclut les personnes dont le repos hebdomadaire ne coïncide pas avec le samedi et le dimanche). La formule d' « horaires atypiques » employée pour désigner tout type d'horaire s'écartant de la semaine standard, serait donc impropre, si l'on

\section{Encadré 2}

\section{SIX TYPES DE SEMAINES DE TRAVAIL}

Une journée étant considérée comme travaillée si elle compte plus d'un quart d'heure de travail, et définie comme pleine si elle en comporte au moins cinq heures (1), on définit six types de semaine de travail, distingués sur la base des informations disponibles dans le semainier :

- Semaine avec travail de nuit : au moins cinq heures (consécutives ou non) du travail de la semaine sont effectuées de $23 \mathrm{~h}$ à $5 \mathrm{~h}$; ce critère prime sur les suivants (cf. graphique : infirmière).

- Semaine comportant au moins six jours de travail ('agriculteur du graphique relève de ce type).

- Semaine standard: correspondant à des horaires réguliers diurnes et à une durée du travail proche de la moyenne, elle est définie par le cumul de quatre critères :

- deux jours de repos consécutifs,

- cinq jours de travail pleins (cinq heures de travail au moins),
- horaire entièrement effectué entre $5 \mathrm{~h}$ et $23 \mathrm{~h}$ (pas de travail de nuit),

- horaire hebdomadaire compris entre 35 et $44 \mathrm{~h}$.

Pour $82 \%$ des semaines standard, les deux jours de repos sont constitués par le samedi et le dimanche ('’ingénieur du graphique relève de ce type).

- Semaine de cinq jours, sauf semaine standard (voir le professeur du graphique).

- Semaine de quatre jours de travail.

- Autres semaines (principalement trois jours de travail).

1. Parmi les journées composant les semaines normales de travail, $27,3 \%$ sont de durée nulle, 0,9\% de durée non nulle inférieure à trois heures, 2,8 \% de durée inférieure à cinq heures et supérieure ou égale à trois heures.

\section{Quatre exemples de semainier}

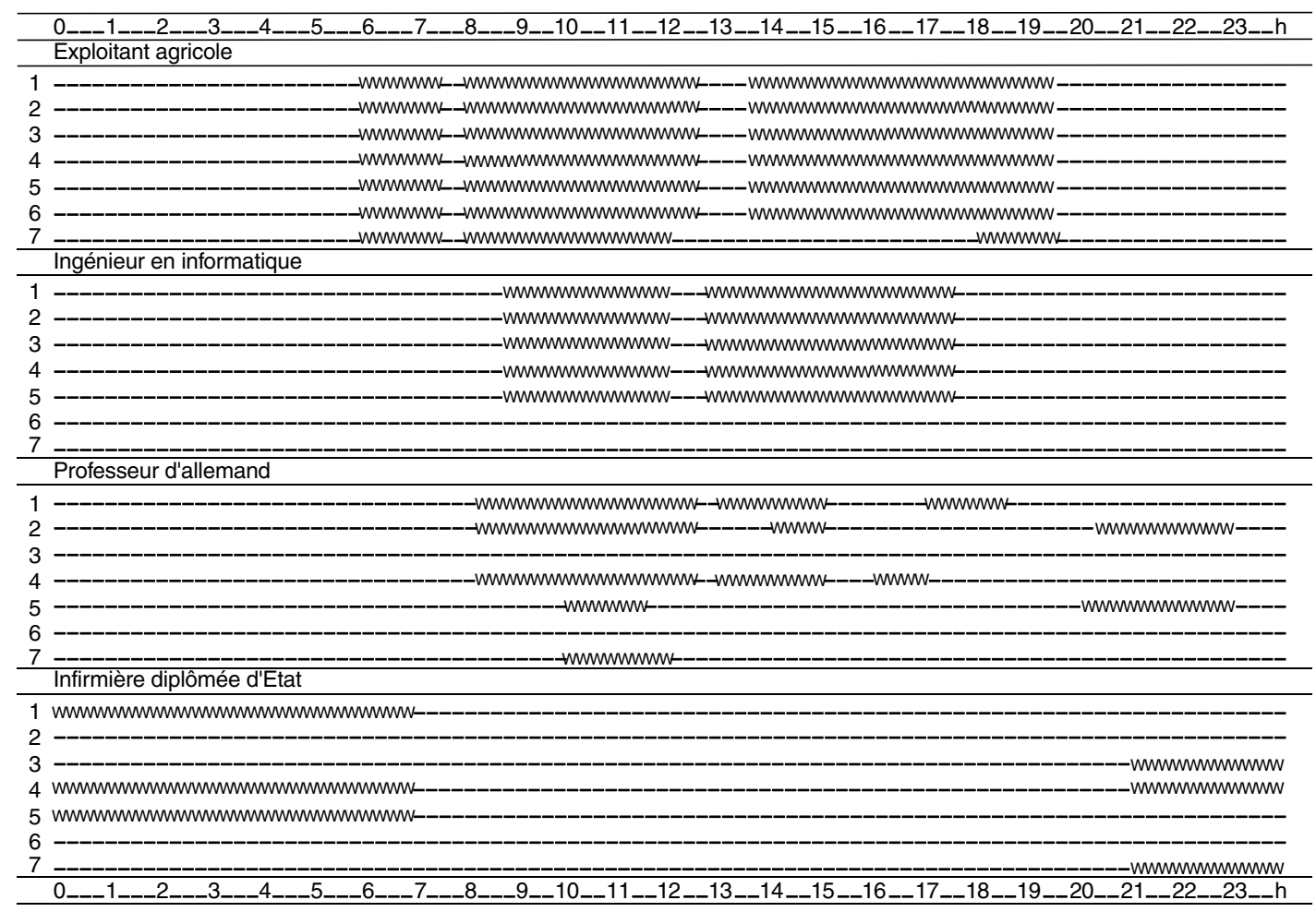

Lecture : une ligne représente une journée décomposée en 96 quarts d'heure, marqués " $W$ » en cas d'activité professionnelle. Sept lignes, numérotées de 1 (lundi) à 7 (dimanche), décrivent une semaine. 
considère que la semaine standard n'est qu'un type d'horaire - nettement minoritaire - parmi d'autres.

\section{Graphique 1}

\section{Horaires quotidiens des cadres et des salariés} peu qualifiés

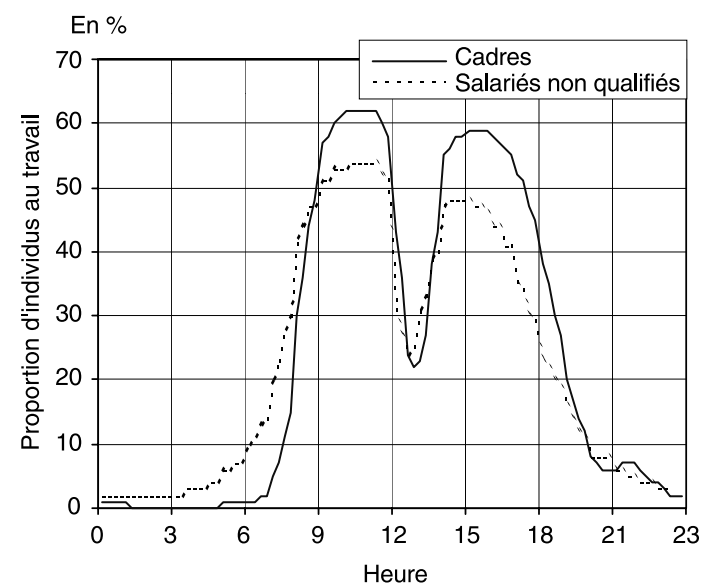

Lecture : la proportion d'individus au travail figure en ordonnées. Les employés et ouvriers non qualifiés sont nombreux à travailler tôt le matin. Les horaires de travail des cadres, plus lourds, se concentrent davantage sur les tranches du matin $(9 h-12 h)$ et de l'après-midi (14 h-19 h). Les courbes des professions intermédiaires et des employés et ouvriers qualifiés, qui n'ont pas été reproduites, s'étagent entre les deux précédentes.

Champ: salariés à temps plein dont le semainier décrit une semaine de travail normale.

Source : enquête Emploi du temps, 1998-1999, Insee.
La semaine standard caractérise les grandes organisations bureaucratiques; elle est un « horaire de bureau », plus qu'un « horaire de fonctionnaire », puisqu'elle est beaucoup plus répandue dans les grands établissements du secteur privé que dans la fonction publique (45\% et $30 \%$ respectivement - cf. tableau 2). Elle est particulièrement fréquente chez les employés et ouvriers qualifiés, ainsi que chez les titulaires d'un CAP ou d'un BEP. Elle ne concerne que $3 \%$ des non-salariés.

Les semaines de cinq jours non standard touchent pratiquement toutes les professions, mais sont particulièrement fréquentes chez les salariés hautement qualifiés. Elles concernent un quart des actifs à temps plein.

La semaine de quatre jours touche un peu plus d'un actif à temps plein sur dix. Maximale dans la fonction publique (15\%), sa fréquence diminue avec la taille de l'établissement et atteint son minimum chez les non salariés (5\%).

Inversement, les semaines sans jour de repos ou n'en comptant qu'un (semaines de six jours ou plus) sont très majoritaires chez les non salariés, fréquentes chez les salariés des très petites entreprises, et rares parmi les salariés des grands établissements. Ces semaines longues concernent un quart des actifs occupés à temps plein.

Tableau 2

Types de semaine et caractéristiques individuelles

\begin{tabular}{|c|c|c|c|c|c|c|c|}
\hline & \multirow{2}{*}{$\begin{array}{l}\text { Part du } \\
\text { week-end } \\
\text { (1) }\end{array}$} & \multicolumn{6}{|c|}{ Type de semaine } \\
\hline & & $\begin{array}{c}\text { Avec travail } \\
\text { de nuit }\end{array}$ & $\begin{array}{c}6 \text { ou } \\
7 \text { jours }\end{array}$ & $\begin{array}{l}5 \text { jours } \\
\text { standard }\end{array}$ & $\begin{array}{l}5 \text { jours non } \\
\text { standard }\end{array}$ & 4 jours & $\begin{array}{c}3 \text { jours } \\
\text { ou moins }\end{array}$ \\
\hline $\begin{array}{l}\text { Homme } \\
\text { Femme }\end{array}$ & $\begin{array}{r}9,4 \\
10,3\end{array}$ & $\begin{array}{l}7,3 \\
3,1\end{array}$ & $\begin{array}{l}24,6 \\
25,0\end{array}$ & $\begin{array}{l}30,8 \\
34,7\end{array}$ & $\begin{array}{l}25,0 \\
21,4\end{array}$ & $\begin{array}{r}9,4 \\
12,3\end{array}$ & $\begin{array}{l}2,9 \\
3,5\end{array}$ \\
\hline $\begin{array}{l}\text { Moins de } 30 \text { ans } \\
30 \text { à } 49 \text { ans } \\
50 \text { ans et plus }\end{array}$ & $\begin{array}{r}10,1 \\
9,5 \\
10,1\end{array}$ & $\begin{array}{l}7,4 \\
5,5 \\
5,1\end{array}$ & $\begin{array}{l}17,8 \\
24,7 \\
31,7\end{array}$ & $\begin{array}{l}36,2 \\
33,0 \\
25,9\end{array}$ & $\begin{array}{l}24,2 \\
23,2 \\
24,7\end{array}$ & $\begin{array}{r}11,2 \\
10,7 \\
9,1\end{array}$ & $\begin{array}{l}3,2 \\
2,9 \\
3,6\end{array}$ \\
\hline $\begin{array}{l}\text { Sans diplôme, CEP, sans réponse } \\
\text { CAP, BEP } \\
\text { Baccalauréat au plus } \\
\text { Diplôme supérieur au baccalauréat }\end{array}$ & $\begin{array}{r}10,7 \\
10,1 \\
9,1 \\
8,7\end{array}$ & $\begin{array}{l}7,0 \\
6,4 \\
6,7 \\
3,4\end{array}$ & $\begin{array}{l}26,0 \\
22,8 \\
23,2 \\
27,8\end{array}$ & $\begin{array}{l}34,2 \\
33,6 \\
34,3 \\
27,2\end{array}$ & $\begin{array}{l}19,1 \\
22,8 \\
21,0 \\
30,1\end{array}$ & $\begin{array}{r}9,9 \\
11,3 \\
12,3 \\
8,6\end{array}$ & $\begin{array}{l}3,9 \\
3,0 \\
2,4 \\
2,9\end{array}$ \\
\hline $\begin{array}{l}\text { Salarié de la Fonction publique } \\
\text { Salarié d'un grand étab. (plus de } 500 \text { sal.) } \\
\text { Salarié d'un étab. de } 10 \text { à } 499 \text { salariés } \\
\text { Salarié petit établ. (moins de } 10 \text { sal.) } \\
\text { Non salarié }\end{array}$ & $\begin{array}{r}9,2 \\
7,2 \\
7,9 \\
10,9 \\
17,0\end{array}$ & $\begin{array}{l}7,0 \\
7,7 \\
5,8 \\
4,1 \\
3,9\end{array}$ & $\begin{array}{l}22,3 \\
12,0 \\
13,9 \\
29,2 \\
69,0\end{array}$ & $\begin{array}{r}30,3 \\
44,6 \\
38,3 \\
32,3 \\
3,1\end{array}$ & $\begin{array}{l}21,5 \\
19,9 \\
28,4 \\
23,4 \\
17,9\end{array}$ & $\begin{array}{r}14,6 \\
12,0 \\
10,2 \\
8,9 \\
5,0\end{array}$ & $\begin{array}{l}4,3 \\
3,8 \\
3,4 \\
2,0 \\
1,0\end{array}$ \\
\hline $\begin{array}{l}\text { Cadres et assimilés } \\
\text { Professions intermédiaires } \\
\text { Employés et ouvriers qualifiés } \\
\text { Employés et ouvriers non qualifiés }\end{array}$ & $\begin{array}{l}6,3 \\
8,7 \\
8,7 \\
9,6\end{array}$ & $\begin{array}{l}1,9 \\
4,9 \\
7,9 \\
6,6\end{array}$ & $\begin{array}{l}25,5 \\
20,0 \\
11,9 \\
20,0\end{array}$ & $\begin{array}{l}20,9 \\
35,9 \\
44,8 \\
34,6\end{array}$ & $\begin{array}{l}39,9 \\
24,1 \\
18,8 \\
25,2\end{array}$ & $\begin{array}{r}8,7 \\
11,7 \\
12,8 \\
10,4\end{array}$ & $\begin{array}{l}3,1 \\
3,4 \\
3,8 \\
3,2\end{array}$ \\
\hline Ensemble & 9,7 & 5,8 & 24,8 & 32,2 & 23,7 & 10,5 & 3,1 \\
\hline
\end{tabular}

Lecture : 32,2 \% des actifs occupés à temps plein ont un horaire hebdomadaire de travail du type " semaine standard ». Pour la définition des types d'horaire, se reporter à l'encadré 2.

Champ : actifs occupés à temps plein et décrivant une semaine normale de travail.

Source : enquête Emploi du temps, 1998-1999, Insee. 
Les semaines avec travail de nuit concernent les hommes plus que les femmes, les jeunes plus que les plus âgés, les titulaires de diplômes de tous niveaux sauf ceux supérieurs au baccalauréat, les salariés de la Fonction publique ou des grands établissements plutôt que des petits établissements, les salariés d'exécution plutôt que les cadres.

\section{Le travail à domicile s'accompagne d'horaires moins réguliers}

La variabilité des horaires d'une semaine sur l'autre pour un même individu n'est pas observée directement : les répondants indiquent une fourchette en réponse à la question «Quelle durée effective en heures travaillez-vous habituellement chaque semaine: Minimum ?
Maximum ?». La fourchette est plus ouverte chez les hommes que chez les femmes, chez les non salariés que chez les salariés, chez les très diplômés que chez les peu diplômés (cf. tableau 3, dernière colonne). La variabilité interindividuelle peut, elle, être observée directement. L'écart-type de la durée du travail observée au semainier (cf. tableau 1) présente des variations dans l'ensemble similaires à celles des fourchettes individuelles.

La semaine standard va de pair avec des horaires réguliers d'une semaine sur l'autre : c'est pour ce type de semaine que l'écart entre l'horaire hebdomadaire le plus lourd et le plus léger est minimum (cf. graphique II). Un lieu de travail fixe et distinct du domicile est aussi un facteur de régularité des horaires et est associé à une fréquence plus élevée de la semaine stan-

Tableau 3

\section{Représentations du temps de travail}

\begin{tabular}{|c|c|c|c|c|c|c|c|c|c|c|}
\hline & $\begin{array}{l}\text { Fati- } \\
\text { gué }\end{array}$ & $\begin{array}{c}\text { Débor- } \\
\text { dé }\end{array}$ & $\begin{array}{c}\text { Manque } \\
\text { temps } \\
\text { trav. }\end{array}$ & $\begin{array}{c}\text { Libre } \\
\text { pauses }\end{array}$ & $\begin{array}{l}\text { Libre } \\
\text { absen- } \\
\text { ces (1) }\end{array}$ & $\begin{array}{l}\text { Heures } \\
\text { suppl. } \\
\text { (1) }\end{array}$ & $\begin{array}{l}\text { Prolonge } \\
\text { journée } \\
(1)\end{array}$ & $\begin{array}{c}\text { Travail } \\
\text { à } \\
\text { domicile }\end{array}$ & $\begin{array}{c}\text { Part } \\
\text { trav.à } \\
\text { domicile }\end{array}$ & $\begin{array}{l}\text { Four- } \\
\text { chette }\end{array}$ \\
\hline $\begin{array}{l}\text { Homme } \\
\text { Femme }\end{array}$ & $\begin{array}{l}42,6 \\
42,8\end{array}$ & $\begin{array}{l}18,9 \\
23,3\end{array}$ & $\begin{array}{l}31,7 \\
33,9\end{array}$ & $\begin{array}{l}72,0 \\
62,4\end{array}$ & $\begin{array}{l}26,5 \\
20,1\end{array}$ & $\begin{array}{l}37,1 \\
27,2\end{array}$ & $\begin{array}{l}46,1 \\
52,0\end{array}$ & $\begin{array}{l}19,4 \\
22,0\end{array}$ & $\begin{array}{l}6,5 \\
9,0\end{array}$ & $\begin{array}{l}14,3 \\
12,2\end{array}$ \\
\hline $\begin{array}{l}\text { Moins de } 30 \text { ans } \\
30 \text { à } 49 \text { ans } \\
50 \text { ans et plus }\end{array}$ & $\begin{array}{l}39,6 \\
42,7 \\
45,5\end{array}$ & $\begin{array}{l}16,2 \\
22,6 \\
18,3\end{array}$ & $\begin{array}{l}26,2 \\
34,0 \\
34,0\end{array}$ & $\begin{array}{l}57,4 \\
70,8 \\
73,1\end{array}$ & $\begin{array}{l}19,1 \\
25,6 \\
25,1\end{array}$ & $\begin{array}{l}37,9 \\
34,6 \\
26,4\end{array}$ & $\begin{array}{l}53,2 \\
48,8 \\
41,6\end{array}$ & $\begin{array}{l}16,8 \\
20,8 \\
22,3\end{array}$ & $\begin{array}{l}3,8 \\
7,7 \\
9,9\end{array}$ & $\begin{array}{l}13,4 \\
13,3 \\
14,6\end{array}$ \\
\hline $\begin{array}{l}\text { Sans diplôme, CEP, sans réponse } \\
\text { CAP, BEP } \\
\text { Baccalauréat au plus } \\
\text { Diplôme supérieur au baccalauréat }\end{array}$ & $\begin{array}{l}43,0 \\
41,7 \\
43,5 \\
43,5\end{array}$ & $\begin{array}{l}15,3 \\
18,1 \\
19,8 \\
28,8\end{array}$ & $\begin{array}{l}22,4 \\
30,7 \\
31,5 \\
43,6\end{array}$ & $\begin{array}{l}61,6 \\
69,4 \\
72,0 \\
71,1\end{array}$ & $\begin{array}{l}15,6 \\
21,3 \\
30,1 \\
32,5\end{array}$ & $\begin{array}{l}33,5 \\
36,7 \\
33,5 \\
28,6\end{array}$ & $\begin{array}{l}30,0 \\
43,2 \\
50,7 \\
69,0\end{array}$ & $\begin{array}{r}3,6 \\
11,3 \\
20,5 \\
47,5\end{array}$ & $\begin{array}{r}6,1 \\
5,1 \\
6,3 \\
12,3\end{array}$ & $\begin{array}{l}10,1 \\
12,9 \\
13,9 \\
17,2\end{array}$ \\
\hline $\begin{array}{l}\text { Salarié de la Fonction publique } \\
\text { Salarié d'un grand étab. (plus de } 500 \text { sal.) } \\
\text { Salarié d'un étab. de } 10 \text { à } 499 \text { salariés } \\
\text { Salarié petit établ. (moins de } 10 \text { sal.) } \\
\text { Non salarié }\end{array}$ & $\begin{array}{l}36,3 \\
36,0 \\
40,5 \\
43,1 \\
67,0\end{array}$ & $\begin{array}{l}18,7 \\
18,2 \\
18,4 \\
20,8 \\
31,7\end{array}$ & $\begin{array}{l}29,6 \\
30,5 \\
30,5 \\
33,1 \\
44,7\end{array}$ & $\begin{array}{l}60,0 \\
75,0 \\
64,9 \\
68,5 \\
88,1\end{array}$ & $\begin{array}{l}21,9 \\
31,9 \\
29,6 \\
26,6 \\
/ /\end{array}$ & $\begin{array}{l}33,5 \\
43,1 \\
43,1 \\
29,6 \\
/ /\end{array}$ & $\begin{array}{c}63,3 \\
52,1 \\
52,1 \\
53,3 \\
/ /\end{array}$ & $\begin{array}{r}39,3 \\
24,7 \\
19,2 \\
11,9 \\
0,0\end{array}$ & $\begin{array}{r}12,4 \\
3,1 \\
3,6 \\
6,9 \\
14,5\end{array}$ & $\begin{array}{l}15,0 \\
10,5 \\
10,9 \\
12,8 \\
23,9\end{array}$ \\
\hline $\begin{array}{l}\text { Cadres et assimilés } \\
\text { Professions intermédiaires } \\
\text { Employés et ouvriers qualifiés } \\
\text { Employés et ouvriers non qualifiés }\end{array}$ & $\begin{array}{l}44,0 \\
39,6 \\
33,9 \\
43,5\end{array}$ & $\begin{array}{l}30,0 \\
23,6 \\
14,8 \\
12,2\end{array}$ & $\begin{array}{l}46,0 \\
38,4 \\
25,4 \\
20,1\end{array}$ & $\begin{array}{l}77,1 \\
71,8 \\
63,5 \\
54,5\end{array}$ & $\begin{array}{l}54,3 \\
34,1 \\
20,9 \\
16,0\end{array}$ & $\begin{array}{l}35,3 \\
35,6 \\
43,3 \\
37,1\end{array}$ & $\begin{array}{l}86,8 \\
72,6 \\
43,2 \\
39,4\end{array}$ & $\begin{array}{r}67,7 \\
41,1 \\
7,5 \\
3,1\end{array}$ & $\begin{array}{r}15,5 \\
6,3 \\
1,7 \\
7,1\end{array}$ & $\begin{array}{r}18,3 \\
14,4 \\
9,1 \\
10,1\end{array}$ \\
\hline Ensemble & 42,7 & 20,5 & 32,5 & 68,6 & 24,2 & 33,6 & 48,2 & 20,3 & 7,4 & 13,6 \\
\hline
\end{tabular}

Lecture: dans les colonnes 2 à 9 figure la proportion d'enquêtés ayant répondu par l'affirmative aux questions relatives à la représentation du temps de travail par les intéressés, dont l'abréviation figure en intitulé de colonne. La signification de ces abréviations est la suivante : Fatigué : à la fin d'une journée normale de travail, se sent fatigué à cause de la durée de la journée de travail.

Débordé : se sent souvent débordé.

Manque temps trav. : manque de temps au travail

Libre pauses : peut interrompre son travail en dehors des pauses éventuelles prévues.

Libre absences : peut sans difficulté s'absenter plusieurs heures pendant le travail.

Heures suppl. : effectue des heures supplémentaires ou complémentaires rémunérées.

Prolonge journée : prolonge sa journée de travail, en dehors des heures supplémentaires ou complémentaires rémunérées.

Travail à domicile : rapporte du travail à faire à la maison (souvent, quelquefois ou rarement).

Ainsi, $30 \%$ des cadres et assimilés se sentent souvent débordés.

Dans les deux dernières colonnes figurent deux indicateurs relatifs à l'organisation individuelle du travail :

Part trav.à domicile : part du travail professionnel effectué à domicile (source : carnet d'activités).

Fourchette: horaires estimés, fourchette d'estimation. Le questionnaire individuel de l'enquête de 1998-1999 comporte les questions suivantes: "Quelle durée effective en heures travaillez-vous habituellement chaque semaine: Minimum ? Maximum ? ". HHMIN et HHMAX étant les deux réponses et HEST leur moyenne arithmétique, la fourchette, définie comme FOUR = $100 \times(\mathrm{HHMAX}-\mathrm{HHMIN}) /$ HEST, constitue un indicateur de variabilité des horaires d'une semaine sur l'autre.

Ainsi, les cadres effectuent 15,5\% de leur travail personnel à domicile, et la variation des horaires hebdomadaires d'une semaine sur l'autre est maxiimum pour les non-salariés (fourchette de 23,9\%).

Champ : actifs occupés à temps plein et décrivant une semaine normale de travail.

Source : enquête Emploi du temps, 1998-1999, Insee. 
dard, tandis que le travail à domicile et le travail itinérant sont fréquemment associés à des horaires en dehors des plages quotidiennes habituelles (cf. graphique III). Dans le premier cas, le contrôle de l'activité du salarié revient en général à vérifier sa présence sur le lieu de travail. Dans le second il fait plutôt l'objet d'une évaluation du résultat atteint, indépendamment des dispositions qu'il a pu prendre pour organiser son temps.

\section{Les cadres sont plus nombreux à se sentir débordés}

Se sentir fatigué en fin de journée en raison de la durée de la journée de travail est très fréquent chez les non salariés, fréquent chez les cadres et les salariés les moins qualifiés, et relativement rare chez les employés et ouvriers qualifiés.

$23 \%$ des femmes - mais seulement $19 \%$ des hommes - se sentent souvent ou très souvent débordées. Les titulaires d'un diplôme supérieur au baccalauréat, et, dans une moindre mesure, les non salariés et les cadres sont plus nombreux à être dans ce cas. Le sentiment d'être débordé (de manière générale) et celui de manquer de temps au travail sont d'autant plus répandus que

\section{Graphique II \\ Type de semaine, travail à domicile, variabilité des horaires}

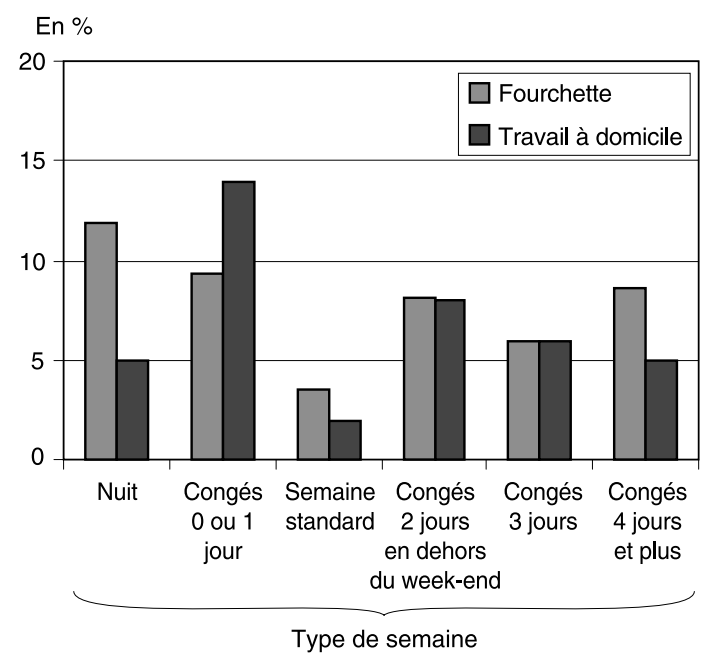

Lecture : la fourchette est un indicateur d'écart entre le minimum et le maximum de l'horaire habituel (se reporter au tableau 3), l'indicateur de travail à domicile donne la part de l'horaire de travail effectué à domicile, mesurée à partir du carnet quotidien d'activités. Les actifs effectuant une semaine standard (cf. encadré 2) ont des horaires qui varient peu d'une semaine habituelle à une autre ; $2 \%$ seulement environ de leurs horaires de travail prennent place à domicile.

Champ : actifs à temps plein ayant décrit une semaine de travail normale à leur semainier.

Source : enquête Emploi du temps, 1998-1999, Insee. les niveaux de formation et de qualification sont élevés.

\section{L'autonomie des plus qualifiés va de pair avec de plus longues journées de travail}

Les indicateurs d'autonomie dans l'organisation du temps de temps de travail s'ordonnent de

\section{Graphique III}

Horaires du travail professionnel effectué par les salariés

\section{A - Sur leur lieu de travail}

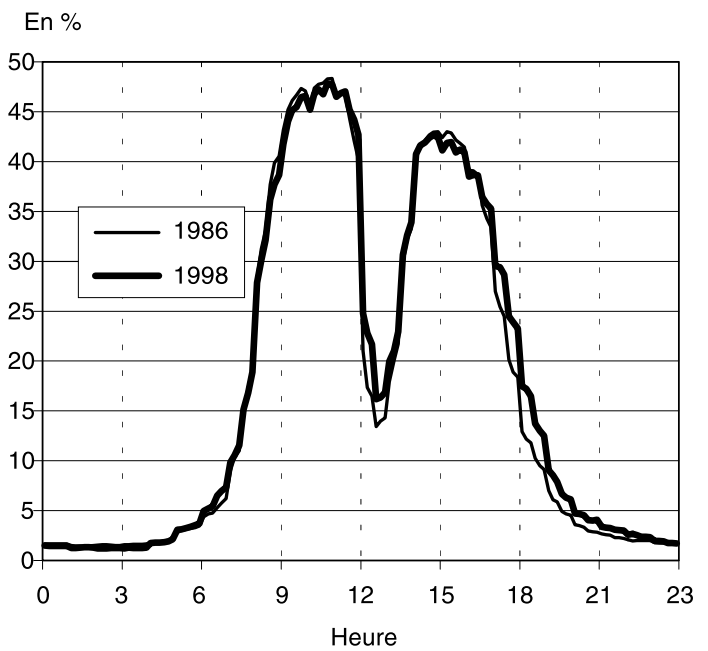

\section{B - À domicile}

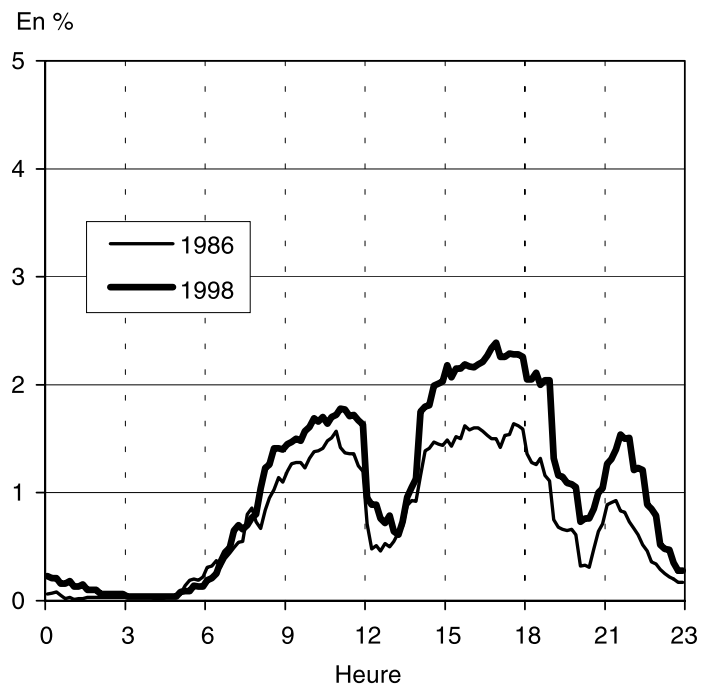

Lecture : Sur le lieu de travail habituel (fixe et distinct du domicile), le travail s'effectue surtout de $9 \mathrm{~h}$ à midi et de $14 \mathrm{~h}$ à $17 \mathrm{~h}$. c'est vers $10 \mathrm{~h} 30$ que la proportion de personnes occupées est la plus élevée (48\%). À domicile, les plages sont plus larges et la courbe présente trois modes dont le plus élevé $(2,3 \%$ en 1998) est atteint vers $17 \mathrm{~h}$. La fréquence du travail à domicile s'accroît sensiblement de 1986 à 1998.

Champ : salariés à temps plein ayant fourni un carnet quotidien d'activités.

Source : enquête Emploi du temps, 1998-1999, Insee. 
la même manière : les ouvriers et employés peu qualifiés sont moins nombreux que les cadres à déclarer pouvoir interrompre leur travail en dehors des pauses éventuelles prévues, à pouvoir s'absenter plusieurs heures pendant leur travail, les scores des employés et ouvriers qualifiés et des membres des professions intermédiaires s'étageant entre ceux des deux premières catégories. Les salariés des grands établissements bénéficient de plus de liberté dans le choix de leurs dates de vacances et dans l'organisation de leurs temps de pause. Les femmes, plus nombreuses que les hommes à occuper des emplois peu ou pas qualifiés, sont moins nombreuses à se déclarer libres de faire des pauses ou de s'absenter du travail. L'autonomie des plus qualifiés et des plus diplômés semble aller de pair avec une plus grande propension à prolonger les journées de travail au-delà de leur durée habituelle, et avec un moindre cloisonnement entre vie professionnelle et vie domestique.

La pratique des heures supplémentaires ou complémentaires - décomptées par l'employeur et rémunérées de manière non forfaitaire - est caractéristique des employés et des ouvriers qualifiés.

\section{Six types d'organisation du temps de travail}

$\mathbf{O}$ n utilise dans ce qui suit une nomenclature socioprofessionnelle plus détaillée (cf. encadré 3) (cf. tableaux 4 à 6). Les caractéristiques décrites (répartition selon sept tranches horaires et six types de semaine, sentiments d'être débordé et d'être plus ou moins libre d'organiser son temps décrits au travers des variables du tableau 6 à l'exception de celles qui ne concernent que les salariés) servent de base à plusieurs analyses des données (analyse en composantes principales, classification ascendante hiérarchique) (2). Elles font apparaître six groupes de catégories socioprofessionnelles au

2. Dans ces analyses, les unités élémentaires sont les catégories socioprofessionnelles détaillées des tableaux 4 à 6 , pondérés par leur effectif.

Encadré 3

\section{UNE NOMENCLATURE SOCIOPROFESSIONNELLE SPÉCIFIQUE}

La nomenclature professionnelle retenue est celle des catégories socioprofessionnelles à deux chiffres (Insee, 1992), aux modifications suivantes près, qui visent à isoler, lorsque les effectifs le permettent, des professions dont le temps de travail présente des particularités :

- Les agriculteurs forment une seule catégorie (dans le cadre d'une enquête de ce type, les critères de taille de l'exploitation agricole ne sont pas mis en œuvre avec la fiabilité souhaitable).

- Parmi les professeurs et assimilés (CS 34), les enseignants proprement dits (codes PCS 3411, professeurs agrégés et certifiés, et 3415 , enseignants du supérieur) sont isolés des autres catégories, dont les horaires se rapprochent davantage de ceux des autres cadres de la fonction publique (CS 33).

- De la même manière, parmi les instituteurs et assimilés (CS 42), les professions enseignantes (codes 4211, $4215,4221,4224)$ sont distinguées des autres.

- Les informations sur le clergé (CS44) sont omises en raison de la faiblesse de l'effectif observé (les enquêtes Emploi indiquent par ailleurs que cette catégorie est, parmi toutes les CS, celle qui travaille le plus le dimanche).

- Parmi les professions intermédiaires administratives et commerciales des entreprises (CS 42), les représen- tants de commerce (PCS 4624 à 4627), qui n'ont pas de lieu de travail fixe, sont distingués du reste de la catégorie.

- Parmi les employés civils de la fonction publique (CS 52), les employés administratifs et assimilés (52A) sont distingués des agents de services (52B : 5216 à 5222 sauf 5221,5223$)$, moins qualifiés.

- Parmi les personnels des services directes aux particuliers (56), les assistantes maternelles, gardiennes d'enfants et travailleuses familiales (code 5631), qui exercent à leur propre domicile une grande partie de leurs activités professionnelles, sont isolées.

- Parmi les chauffeurs (64), les conducteurs routiers et grands routiers (6411) sont distingués du reste de la catégorie.

Ces rubriques sont ici regroupées, dans le champ des emplois salariés, en sous-ensembles formant une échelle ordonnée selon quatre grands niveaux de formation et de rémunération :

- Cadres et assimilés (CS 33 à 38).

- Professions intermédiaires (CS 42 à 48).

- Employés et ouvriers qualifiés (CS 52A, 53, 54, 62, $63,64 \mathrm{C}, 65)$.

- Employés et ouvriers non qualifiés (52B, 55, 56, 64L, $67,68,69)$. 
sein desquels les horaires et la perception du temps de travail s'avèrent relativement homogènes. Ce découpage est compatible avec le découpage préexistant des salariés en trois pôles : encadrement, pôle industriel, métiers du commerce (Bué et Rougerie, 1999) (3). Il distingue les non-salariés au moyen d'un groupement supplémentaire, tandis que le niveau de description des salariés est un peu plus détaillé: le pôle industriel-bureaucratique est scindé en deux (horaires décalés ou non), les enseignants forment une catégorie distincte, et le pôle des métiers du commerce incorpore certains métiers des services aux personnes.

L'un des axes au long desquels se distribuent les six groupes de professions oppose des salariés d'exécution dont la rémunération est étroitement liée à une mesure assez précise de la durée du travail à des actifs, non salariés ou enseignants, qui disposent à l'inverse d'une grande latitude dans la détermination de leurs horaires de travail. Un second axe, exprimant principalement la durée du travail, oppose les non salariés aux enseignants.

\section{Groupe I : autonomie et horaires lourds pour les entrepreneurs}

Alors que ni le statut d'emploi ni l'appartenance socioprofessionnelle ne figurent parmi les variables actives dans l'analyse des données, l'emploi du temps à lui seul suffit à individualiser les non-salariés. Les entrepreneurs travaillent peu la nuit, beaucoup en fin de journée. Leurs horaires sont lourds, varient fortement d'un individu à un autre: l'écart-type de l'horaire hebdomadaire est sensiblement plus élevé pour les non-salariés $(17,2 \mathrm{~h}$, cf. tableau 1). Ils varient fortement aussi d'une semaine sur l'autre pour un même individu : c'est également pour les non-salariés qu'en moyenne, l'écart entre les horaires extrêmes représente la plus forte proportion de l'horaire moyen, à savoir près de $24 \%$ (cf. tableau 3) (4). Les membres de ces professions apportent souvent du travail à faire à la maison, quand ils ne travaillent pas tout le temps à domicile. Ils sont libres de prendre des pauses à leur convenance. Ils se plaignent de manquer de temps, notamment dans le cadre de leur journée de travail.

L'éventail d'horaires pratiqués dans ce groupe va d'horaires lourds, envahissant tous les jours de la semaine y compris le dimanche, à des rythmes de travail voisins de ceux des salariés et plus étroitement encadrés par le droit du travail (cf. tableau 4). Par ordre de durée du travail décroissante, se succèdent ainsi les agriculteurs, les commerçants, les chefs d'entreprise, les artisans, les membres des professions libérales, les professions de l'information, des arts et des spectacles. Cette dernière catégorie comprend une majorité de salariés, aux horaires moyens assez légers, mais beaucoup de ces salariés décident de leurs horaires comme le font les indépendants. Les membres des professions libérales et des professions de l'information, des arts et des spectacles sont les moins matinaux de tous les actifs travaillant à temps plein: ils n'effectuent que 4 ou $5 \%$ de leurs heures de travail entre $5 \mathrm{~h}$ et $9 \mathrm{~h}$. Un actif à temps plein sur sept relève de ce groupe, qui est, par ailleurs, surtout masculin.

\section{Groupe II : le dualisme heures statutaires/ temps de préparation des enseignants}

Les professions enseignantes forment un sousgroupe de taille réduite, mais aux caractéristiques nettement typées : horaire total de travail assez léger et réparti en nombreux épisodes relativement brefs, se situant pour partie à domicile, à des heures tardives et durant le week-end. Elles sont les seules à présenter une courbe d'activité tri-modale, avec un maximum d'activité le matin de 10 à $11 \mathrm{~h}$, un autre l'après-midi vers $15 \mathrm{~h}$, et un troisième en soirée vers $22 \mathrm{~h}$ (cf. graphique IV). Le semainier permet seulement de deviner que l'activité professionnelle s'organise en deux composantes nettement disjointes, les heures statutaires d'enseignement, dont le rythme, en grande part, s'impose à l'enseignant (y compris, assez souvent, le samedi matin), et le temps de préparation et de corrections, réparti de manière bien plus libre.

Les enseignants estiment pour la plupart avoir peu de liberté dans le choix du calendrier de leurs vacances ou dans la détermination des pauses ou des absences au travail. Ce sentiment de contrainte traduit simplement la manière dont ils perçoivent les questions posées par les enquêteurs, et, partant, la difficulté de distinguer travail statutaire et préparation des cours : lorsqu' on leur demande, par exemple, s'ils peuvent s'absenter pendant leur « travail», ils privilégient dans leur réponse le « travail statutaire

3. Ce découpage s'appuie sur les enquêtes sur les conditions de travail.

4. Ce qui confirme les conclusions tirées de l'enquête Emploi de 1999, suivant lesquelles la lourdeur des horaires des indépendants va de pair avec leur irrégularité (Missègue, 2000). 
en présence des élèves », par rapport à la correction de copies ou à la préparation de cours (5).

Le recours aux heures supplémentaires est très fréquent chez les professeurs de l'enseignement secondaire. La proportion de semaines normales de travail est nettement plus faible parmi les enseignants - notamment chez ceux du primaire - que pour les autres catégories (6). Les professeurs se caractérisent, comme les agriculteurs, par l'ampleur de la fourchette à l'intérieur de laquelle se situent leurs horaires habituels (cf. tableau 6, dernière colonne).

$4 \%$ des actifs à temps plein relèvent de ce groupe, qui se caractérise par une sensible prépondérance de femmes : trois cinquièmes des effectifs.

5. Les frontières du travail non statutaire des enseignants sont par nature floues et les tentatives de mesure de la durée du travail de cette catégorie aboutissent à des résultats qui varient amplement en fonction du mode de questionnement. Le minimum est atteint dans le cadre des enquêtes Emploi, où beaucoup d'enseignants donnent une estimation qui n'inclut que leurs horaires statutaires (voir l'encadré 1). Les enquêtes spécifiques menées au sein du ministère de l'Éducation nationale fournissent des estimations plus élevées si le questionnaire évoque, au titre du temps de travail, le temps consacré à des "recherches personnelles " (Bonnet et Murcia, 1996 ; Guillaume, 2000).

6. On entend par "semaine normale de travail » une semaine déclarée comme telle par la personne interrogée, par opposition aux semaines de congés, chômées ou autres.

Tableau 4

Temps de travail selon la tranche horaire, par regroupements de professions

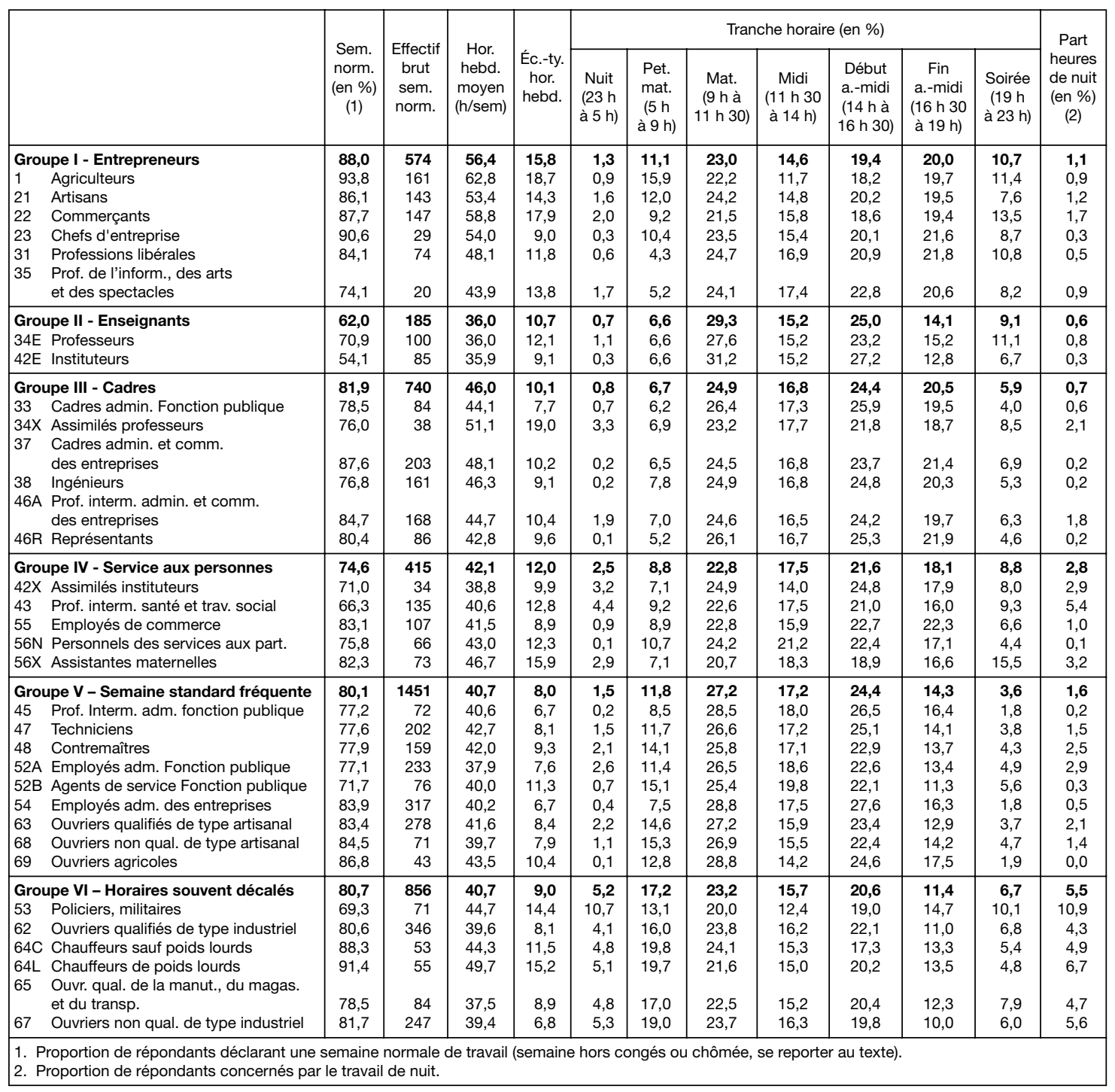

Lecture : dans les colonnes 6 à 12 figurent la répartition par tranche horaire quotidienne des heures hebdomadaires de travail. Ainsi, 11,1\% du travail des professeurs est accompli en soirée, entre 19 h et $23 \mathrm{~h}$. 70,9\% de ces derniers déclarent une semaine de travail normale, et 0,8 \% sont concernés par le travail de nuit. Champ : actifs occupés à temps plein et décrivant une semaine normale de travail.

Source : enquête Emploi du temps, 1998-1999, Insee. 


\section{Groupe III : les débordements d'horaires fréquents mais contrôlés des experts, managers et gestionnaires}

Les ingénieurs, les cadres administratifs des entreprises et de la fonction publique, les cadres commerciaux, les professions intermédiaires administratives et commerciales des entreprises, occupent une position intermédiaire entre les entrepreneurs et les salariés d'exécution. Ils ont une assez grande latitude en matière d'organisation de leur temps de travail. Ils travaillent beaucoup en fin de journée et en soirée, ils sont plus nombreux que les autres salariés à se déclarer débordés et à estimer qu'ils manquent de temps au travail. Il leur arrive fréquemment de faire déborder leur vie professionnelle sur leur temps familial et domestique en effectuant certaines de leurs tâches à domicile, mais dans l'ensemble ils sont moins nombreux que les autres salariés à travailler la nuit ou durant le week-end.

Un actif à temps plein sur six relève de ce groupe, qui compte sept hommes pour trois femmes.

\section{Graphique IV \\ Les professeurs, les employés administratifs d'entreprise et les employés de commerce ont des horaires quotidiens contrastés}
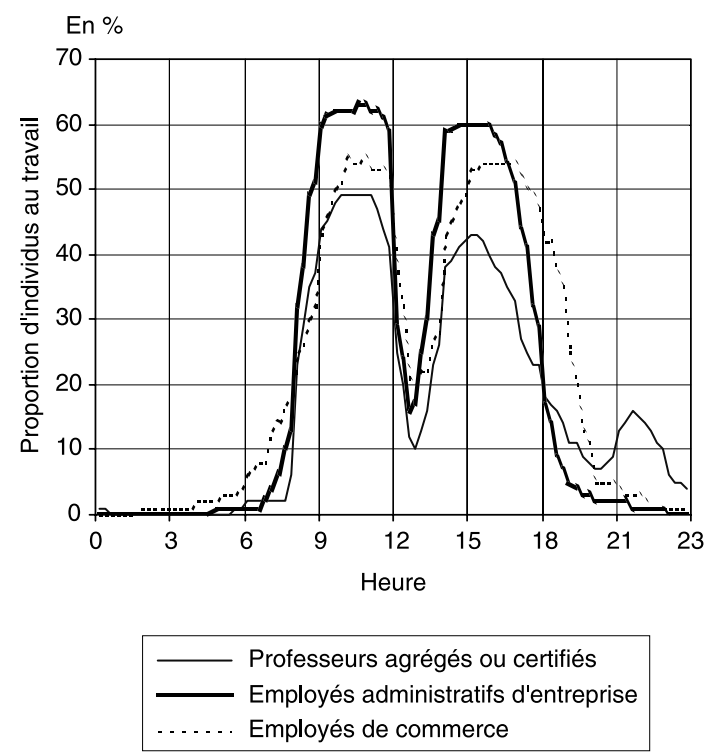

Lecture : la courbe des professeurs présente un mode entre 10 et $11 \mathrm{~h}$, un autre vers $15 \mathrm{~h}$, et un troisième vers $22 \mathrm{~h}$. Celle des employés administratifs d'entreprise fait apparaitre une forte concentration entre $9 \mathrm{~h}$ et $12 \mathrm{~h}$ et entre $14 \mathrm{~h}$ et $16 \mathrm{~h} 30$, alors que les employés de commerce sont assez nombreux à travailler tôt le matin et en soirée.

Champ : actifs à temps plein

Source : enquête Emploi du temps, 1998-1999, Insee.

\section{Groupe IV : les horaires flexibles des professions des services aux personnes}

Les membres des professions intermédiaires de la santé et du travail social (infirmières notamment), les personnels des services directs aux particuliers, les employés de commerce, quelques professions para-enseignantes de niveau intermédiaires (formateurs d'adultes, éducateurs sportifs, etc.) ont souvent des horaires irréguliers, soumis à une certaine flexibilité en fonction des fluctuations de la demande de la clientèle, des patients ou des administrés. Les agents de nettoyage travaillent au petit matin, les serveurs de la restauration font «le coup de chaud » à midi et le soir pendant que les autres professions ont cessé le travail. Les employés de commerce sont nombreux à être au travail en fin d'après-midi et en début de soirée, au moment où la clientèle vient, elle, de finir sa journée (cf. graphique IV). Dans les professions féminines, la palme en matière de travail de nuit revient aux infirmières. Cette profession requérant un niveau de qualification élevé, le taux de travail de nuit des femmes est proportionnel à leur niveau de qualification, alors que c'est l'inverse pour celui des hommes.

Un actif à temps plein sur dix relève de ce groupe, qui est très majoritairement féminin.

\section{Groupe $\mathrm{V}$ : la semaine régulière des agents des grandes organisations bureaucratiques}

Employés administratifs, ouvriers de type artisanal, professions intermédiaires de la fonction publique, techniciens et agents de maîtrise forment un ensemble au sein duquel la semaine standard de cinq jours de travail consécutifs avec horaire diurne régulier est fortement représentée. La semaine de quatre jours est aussi plus répandue que dans les autres professions. $\mathrm{La}$ tranche horaire de fin d'après-midi est sousreprésentée, à l'inverse de ce qu'on observe chez des salariés plus qualifiés. La séparation entre vie professionnelle et autres temps sociaux est bien tranchée : le travail à domicile est rare.

La catégorie la plus emblématique au sein de ce groupe est celle des employés administratifs des entreprises (secrétaires, employés des services comptables et financiers, etc.) : $57 \%$ d'entre eux sont concernés par des horaires hebdomadaires standard, ce qui représente la valeur maximale de cette proportion (cf. tableau 5). La variabilité du nombre d'heures travaillées d'une semaine à l'autre compte parmi les plus faibles. 
$35 \%$ des actifs à temps plein relèvent de ce groupe, qui compte trois hommes pour une femme.

\section{Groupe VI : les horaires décalés des policiers, des militaires et de certains ouvriers}

Les chauffeurs, les ouvriers de l'industrie, les ouvriers de la manutention, du magasinage et du transport sont nombreux à effectuer du travail de nuit, souvent organisé en longues plages horaires regroupées sur un petit nombre de jours de la semaine. Les policiers et militaires se rattachent à ce groupe, mais leurs horaires sont particulièrement irréguliers : l'indicateur d'écart entre leurs horaires habituels les plus faibles et les plus élevés est le plus grand des professions salariées (24\%) (cf. tableau 6). Pour l'importance du travail de nuit ils se situent nettement en tête, devant les salariés d'exécution de l'industrie, alors que ceux-ci gardent l'avantage en matière de travail tôt le matin. Beaucoup de policiers et de militaires déclarent prolonger fréquemment leur journée de travail, mais rares sont ceux qui effectuent des heures supplémentaires ou complémentaires rémunérées.

Tableau 5

Types de semaine de travail par groupe de professions

\begin{tabular}{|c|c|c|c|c|c|c|c|}
\hline & \multirow{2}{*}{$\begin{array}{l}\text { Part du } \\
\text { week-end } \\
\text { (1) }\end{array}$} & \multicolumn{6}{|c|}{ Type de semaine } \\
\hline & & $\begin{array}{l}\text { Avec } \\
\text { travail } \\
\text { de nuit }\end{array}$ & $\begin{array}{l}6 \text { ou } 7 \\
\text { jours }\end{array}$ & $\begin{array}{l}5 \text { jours } \\
\text { standard }\end{array}$ & $\begin{array}{l}5 \text { jours } \\
\text { non } \\
\text { standard }\end{array}$ & $\begin{array}{c}4 \\
\text { jours }\end{array}$ & $\begin{array}{l}3 \text { jours } \\
\text { ou } \\
\text { moins }\end{array}$ \\
\hline $\begin{array}{l}\text { Groupe I - Entrepreneurs } \\
1 \text { Agriculteurs } \\
21 \text { Artisans } \\
22 \text { Commerçants } \\
23 \text { Chefs d'entreprise } \\
31 \text { Professions libérales } \\
35 \text { Prof. de l'inform., des arts et des spectacles }\end{array}$ & $\begin{array}{l}16,8 \\
21,6 \\
15,0 \\
17,9 \\
11,6 \\
11,7 \\
10,6\end{array}$ & $\begin{array}{l}4,6 \\
4,2 \\
4,9 \\
7,3 \\
0,0 \\
1,4 \\
5,0\end{array}$ & $\begin{array}{l}67,1 \\
87,4 \\
59,4 \\
64,8 \\
62,1 \\
51,4 \\
40,0\end{array}$ & $\begin{array}{r}4,5 \\
1,1 \\
4,2 \\
4,7 \\
3,4 \\
8,1 \\
20,0\end{array}$ & $\begin{array}{r}18,3 \\
4,8 \\
22,4 \\
19,4 \\
20,7 \\
33,8 \\
30,0\end{array}$ & $\begin{array}{r}4,7 \\
2,5 \\
7,0 \\
3,3 \\
10,3 \\
5,4 \\
5,0\end{array}$ & $\begin{array}{l}0,8 \\
0,0 \\
2,1 \\
0,6 \\
3,4 \\
0,0 \\
0,0\end{array}$ \\
\hline $\begin{array}{l}\text { Groupe II - Enseignants } \\
\text { 34E Professeurs } \\
\text { 42E Instituteurs }\end{array}$ & $\begin{array}{l}\mathbf{1 1 , 6} \\
12,8 \\
10,1\end{array}$ & $\begin{array}{l}1,1 \\
2,0 \\
0,0\end{array}$ & $\begin{array}{l}46,7 \\
47,8 \\
45,4\end{array}$ & $\begin{array}{l}\mathbf{4 , 4} \\
3,9 \\
4,9\end{array}$ & $\begin{array}{l}35,5 \\
32,4 \\
39,2\end{array}$ & $\begin{array}{l}9,1 \\
8,9 \\
9,4\end{array}$ & $\begin{array}{l}3,3 \\
5,2 \\
1,1\end{array}$ \\
\hline $\begin{array}{l}\text { Groupe III - Cadres } \\
33 \text { Cadres admin. Fonction publique } \\
34 \mathrm{X} \text { Assimilés professeurs } \\
37 \text { Cadres admin. et comm. des entreprises } \\
38 \text { Ingénieurs } \\
\text { 46A Prof. interm. adm. et comm. des entreprises } \\
\text { 46R Représentants }\end{array}$ & $\begin{array}{r}6,3 \\
4,3 \\
8,0 \\
5,0 \\
3,9 \\
10,3 \\
7,5\end{array}$ & $\begin{array}{r}2,2 \\
2,4 \\
13,2 \\
0,5 \\
0,0 \\
4,8 \\
0,0\end{array}$ & $\begin{array}{l}19,7 \\
15,5 \\
42,1 \\
20,2 \\
17,0 \\
20,7 \\
15,8\end{array}$ & $\begin{array}{l}29,3 \\
36,9 \\
21,1 \\
19,1 \\
26,4 \\
39,9 \\
34,1\end{array}$ & $\begin{array}{l}37,1 \\
36,9 \\
10,5 \\
49,4 \\
43,0 \\
23,8 \\
34,7\end{array}$ & $\begin{array}{r}9,1 \\
6,0 \\
10,5 \\
8,0 \\
10,6 \\
9,0 \\
11,9\end{array}$ & $\begin{array}{l}2,6 \\
2,4 \\
2,6 \\
2,9 \\
3,0 \\
1,7 \\
3,5\end{array}$ \\
\hline $\begin{array}{l}\text { Groupe IV - Service aux personnes } \\
42 X \text { Assimilés instituteurs } \\
43 \text { Prof. interm. santé et trav. social } \\
55 \text { Employés de commerce } \\
56 \mathrm{~N} \text { Personnels des services aux part. } \\
\text { 56X Assistantes maternelles }\end{array}$ & $\begin{array}{r}13,6 \\
6,9 \\
14,0 \\
17,3 \\
5,1 \\
18,2\end{array}$ & $\begin{array}{r}6,8 \\
8,9 \\
11,0 \\
2,7 \\
0,0 \\
10,2\end{array}$ & $\begin{array}{l}31,5 \\
27,4 \\
26,6 \\
33,1 \\
18,9 \\
51,7\end{array}$ & $\begin{array}{r}19,8 \\
19,0 \\
28,4 \\
18,1 \\
16,3 \\
9,9\end{array}$ & $\begin{array}{l}24,6 \\
24,5 \\
12,5 \\
32,0 \\
44,6 \\
18,2\end{array}$ & $\begin{array}{r}\mathbf{1 1 , 9} \\
10,1 \\
12,3 \\
12,4 \\
14,9 \\
8,3\end{array}$ & $\begin{array}{r}5,4 \\
10,1 \\
9,2 \\
1,7 \\
5,4 \\
1,6\end{array}$ \\
\hline $\begin{array}{l}\text { Groupe V - Semaine standard fréquente } \\
45 \text { Prof. interm. de l'adm. et de la Fonction publique } \\
47 \text { Techniciens } \\
48 \text { Contremaîtres } \\
\text { 52A Employés adm. de la Fonction publique } \\
\text { 52B Agents de service de la Fonction publique } \\
54 \text { Employés adm. des entreprises } \\
63 \text { Ouvriers qualifiés de type artisanal } \\
68 \text { Ouvriers non qual. de type artisanal } \\
69 \text { Ouvriers agricoles }\end{array}$ & $\begin{array}{r}8,0 \\
6,9 \\
5,3 \\
8,9 \\
10,6 \\
8,6 \\
7,4 \\
8,3 \\
7,3 \\
7,2\end{array}$ & $\begin{array}{l}3,9 \\
0,0 \\
4,0 \\
6,3 \\
6,7 \\
1,2 \\
1,3 \\
5,4 \\
4,1 \\
0,0\end{array}$ & $\begin{array}{l}14,5 \\
26,0 \\
13,7 \\
14,5 \\
12,8 \\
22,9 \\
12,6 \\
12,0 \\
14,2 \\
25,4\end{array}$ & $\begin{array}{l}45,5 \\
41,4 \\
43,3 \\
40,3 \\
39,8 \\
33,4 \\
56,7 \\
45,5 \\
48,4 \\
48,1\end{array}$ & $\begin{array}{l}19,6 \\
18,2 \\
23,8 \\
23,9 \\
17,1 \\
16,0 \\
14,1 \\
22,8 \\
26,9 \\
15,2\end{array}$ & $\begin{array}{r}\mathbf{1 3 , 6} \\
14,4 \\
14,3 \\
10,7 \\
19,4 \\
20,0 \\
13,1 \\
11,5 \\
3,9 \\
9,1\end{array}$ & $\begin{array}{l}2,8 \\
0,0 \\
0,9 \\
4,4 \\
4,2 \\
6,5 \\
2,3 \\
2,8 \\
2,6 \\
2,0\end{array}$ \\
\hline $\begin{array}{l}\text { Groupe VI - Horaires souvent décalés } \\
53 \text { Policiers, militaires } \\
62 \text { Ouvriers qualifiés de type industriel } \\
64 \text { C Chauffeurs sauf poids lourds } \\
64 \text { L Chauffeurs de poids lourds } \\
65 \text { Ouvr. qual. de la manut., du magas. et du transp. } \\
67 \text { Ouvriers non qual. de type industriel }\end{array}$ & $\begin{array}{r}8,3 \\
17,4 \\
6,1 \\
9,5 \\
6,9 \\
12,0 \\
7,6\end{array}$ & $\begin{array}{r}12,7 \\
36,1 \\
8,7 \\
13,2 \\
16,6 \\
8,7 \\
11,8\end{array}$ & $\begin{array}{r}10,6 \\
11,9 \\
9,4 \\
24,5 \\
17,1 \\
10,5 \\
7,6\end{array}$ & $\begin{array}{l}41,4 \\
22,1 \\
47,3 \\
18,9 \\
19,4 \\
39,7 \\
48,9\end{array}$ & $\begin{array}{l}20,6 \\
15,0 \\
19,6 \\
28,3 \\
33,6 \\
19,2 \\
19,5\end{array}$ & $\begin{array}{r}\mathbf{1 0 , 4} \\
12,1 \\
10,0 \\
11,3 \\
8,3 \\
13,5 \\
9,7\end{array}$ & $\begin{array}{l}\mathbf{4 , 3} \\
2,8 \\
5,0 \\
3,8 \\
5,0 \\
8,3 \\
2,4\end{array}$ \\
\hline
\end{tabular}

Lecture : pour la définition des types de semaine, se reporter à l'encadré 2. 47,8 \% des professeurs ont un horaire hebdomadaire de travail de 6 ou 7 jours. $12,8 \%$ de leur temps travail hebdomadaire est accompli le samedi et le dimanche.

Champ : actifs occupés à temps plein et décrivant une semaine normale de travail.

Source : enquête Emploi du temps, 1998-1999, Insee. 
Un cinquième des actifs à temps plein appartiennent à ce groupe essentiellement masculin.

\section{Des horaires irréguliers entretiennent le sentiment de fatigue et de manque de temps}

Plus l'horaire de travail professionnel (observé au semainier) est lourd, plus élevée est la probabilité que le répondant se déclare débordé, ou fatigué en fin de journée du fait de la durée de la journée de travail effectuée. Cette association, assez logique, n'est qu'une tendance générale (cf. graphique V). À rebours de cette tendance, la perception d'une fatigue liée à la lourdeur des horaires de travail est un peu plus fréquente chez les personnes qui travaillent moins de $35 \mathrm{~h}$ que chez celles qui travaillent 35 ou $40 \mathrm{~h}$. La fréquence du sentiment d'être débordé décline au-delà de $60 \mathrm{~h}$ de travail par semaine (peut-être, au-delà de cette limite, le travail perd-il en intensité ce qu'il gagne en durée).

Tableau 6

Représentations du temps de travail par groupe de professions

En \%

\begin{tabular}{|c|c|c|c|c|c|c|c|c|c|c|}
\hline Groupe & $\begin{array}{l}\text { Fati- } \\
\text { gué }\end{array}$ & $\begin{array}{l}\text { Débor- } \\
\text { dé }\end{array}$ & $\begin{array}{c}\text { Manque } \\
\text { temps } \\
\text { tr. }\end{array}$ & $\begin{array}{c}\text { Libre } \\
\text { pauses }\end{array}$ & $\begin{array}{l}\text { Libre } \\
\text { absen- } \\
\text { ces (1) }\end{array}$ & $\begin{array}{c}\text { Heures } \\
\text { suppl. } \\
\text { (1) }\end{array}$ & $\begin{array}{c}\text { Prolonge } \\
\text { journée } \\
\text { (1) }\end{array}$ & $\begin{array}{c}\text { Travail à } \\
\text { domi- } \\
\text { cile }\end{array}$ & $\begin{array}{l}\text { Part tr.à } \\
\text { domi- } \\
\text { cile }\end{array}$ & $\begin{array}{l}\text { Four- } \\
\text { chette }\end{array}$ \\
\hline $\begin{array}{l}\text { Groupe I - Entrepreneurs } \\
1 \text { Agriculteurs } \\
21 \text { Artisans } \\
22 \text { Commerçants } \\
23 \text { Chefs d'entreprise } \\
31 \text { Professions libérales } \\
35 \text { Prof.de l' information, des arts, } \\
\text { et des spectacles }\end{array}$ & $\begin{array}{l}64,5 \\
65,3 \\
69,2 \\
63,9 \\
58,6 \\
64,9 \\
35,0\end{array}$ & $\begin{array}{l}31,7 \\
20,0 \\
37,1 \\
27,0 \\
48,3 \\
48,6 \\
35,0\end{array}$ & $\begin{array}{l}45,1 \\
34,0 \\
49,0 \\
49,8 \\
55,2 \\
50,0 \\
40,0\end{array}$ & $\begin{array}{l}88,5 \\
96,4 \\
86,0 \\
85,5 \\
96,6 \\
82,4 \\
75,0\end{array}$ & $\begin{array}{l}/ / \\
/ / \\
/ / \\
/ / \\
/ / \\
/ / \\
35,0\end{array}$ & $\begin{array}{l}/ / \\
/ / \\
/ / \\
/ / \\
/ / \\
/ / \\
35,0\end{array}$ & $\begin{array}{l}/ / \\
/ / \\
/ / \\
/ / \\
/ / \\
/ / \\
65,0\end{array}$ & $\begin{array}{r}3,6 \\
0,0 \\
0,0 \\
1,0 \\
17,2 \\
4,1 \\
55,0\end{array}$ & $\begin{array}{r}14,1 \\
15,6 \\
13,7 \\
9,1 \\
4,4 \\
20,8 \\
\\
30,5\end{array}$ & $\begin{array}{l}23,5 \\
29,7 \\
22,3 \\
19,7 \\
18,8 \\
22,2 \\
20,6\end{array}$ \\
\hline $\begin{array}{l}\text { Groupe II - Enseignants } \\
\text { 34E Professeurs } \\
42 \mathrm{E} \text { Instituteurs }\end{array}$ & $\begin{array}{l}31,9 \\
39,0 \\
23,6\end{array}$ & $\begin{array}{l}28,3 \\
30,0 \\
26,3\end{array}$ & $\begin{array}{l}42,7 \\
43,8 \\
41,4\end{array}$ & $\begin{array}{l}16,7 \\
20,8 \\
11,8\end{array}$ & $\begin{array}{l}7,3 \\
8,3 \\
6,2\end{array}$ & $\begin{array}{l}60,8 \\
80,8 \\
37,2\end{array}$ & $\begin{array}{l}81,4 \\
81,7 \\
81,1\end{array}$ & $\begin{array}{l}97,9 \\
98,0 \\
97,7\end{array}$ & $\begin{array}{l}37,4 \\
45,2 \\
28,2\end{array}$ & $\begin{array}{l}22,2 \\
27,9 \\
15,6\end{array}$ \\
\hline $\begin{array}{l}\text { Groupe III - Cadres } \\
33 \text { Cadres admin. Fonction publique } \\
34 \mathrm{X} \text { Assimilés professeurs } \\
37 \text { Cadres admin. et comm. } \\
\text { des entreprises } \\
38 \text { Ingénieurs } \\
46 \mathrm{~A} \text { Prof. interm. admin. et comm. } \\
\text { des entreprises } \\
46 \mathrm{R} \text { Représentants }\end{array}$ & $\begin{array}{l}44,8 \\
38,1 \\
50,0 \\
\\
45,6 \\
48,4 \\
\\
40,4 \\
48,6\end{array}$ & $\begin{array}{l}29,2 \\
21,4 \\
36,8 \\
\\
33,8 \\
26,3 \\
\\
26,7 \\
33,3\end{array}$ & $\begin{array}{l}45,7 \\
40,5 \\
50,0 \\
\\
51,8 \\
42,6 \\
44,7 \\
42,6\end{array}$ & $\begin{array}{l}86,9 \\
91,7 \\
73,7 \\
\\
91,1 \\
88,3 \\
\\
81,5 \\
85,8\end{array}$ & $\begin{array}{l}57,4 \\
60,7 \\
55,3 \\
67,6 \\
63,3 \\
40,1 \\
54,1\end{array}$ & $\begin{array}{l}26,5 \\
20,2 \\
23,7 \\
\\
24,0 \\
30,5 \\
\\
29,6 \\
25,9\end{array}$ & $\begin{array}{l}84,0 \\
88,1 \\
86,8 \\
\\
86,5 \\
90,5 \\
\\
74,4 \\
79,4\end{array}$ & $\begin{array}{r}55,4 \\
64,3 \\
71,1 \\
\\
61,8 \\
57,2 \\
\\
40,4 \\
50,6\end{array}$ & $\begin{array}{r}7,5 \\
4,9 \\
15,0 \\
\\
6,8 \\
8,0 \\
\\
5,1 \\
12,3\end{array}$ & $\begin{array}{r}15,1 \\
13,1 \\
20,9 \\
\\
16,0 \\
16,4 \\
\\
11,9 \\
16,5\end{array}$ \\
\hline $\begin{array}{l}\text { Groupe IV - Service aux personnes } \\
42 X \text { Assimilés instituteurs } \\
43 \text { Prof. interm. santé et trav. social } \\
55 \text { Employés de commerce } \\
56 \mathrm{~N} \text { Personnels des services aux part. } \\
56 \mathrm{X} \text { Assistantes maternelles }\end{array}$ & $\begin{array}{l}50,0 \\
61,8 \\
47,6 \\
49,7 \\
59,4 \\
41,0\end{array}$ & $\begin{array}{l}19,5 \\
36,2 \\
24,2 \\
18,0 \\
10,9 \\
12,8\end{array}$ & $\begin{array}{l}28,9 \\
37,1 \\
35,7 \\
28,6 \\
20,3 \\
20,7\end{array}$ & $\begin{array}{l}53,6 \\
57,1 \\
55,7 \\
40,9 \\
62,1 \\
59,0\end{array}$ & $\begin{array}{l}15,4 \\
24,6 \\
13,8 \\
10,0 \\
14,1 \\
23,1\end{array}$ & $\begin{array}{l}24,6 \\
32,6 \\
19,4 \\
34,8 \\
16,2 \\
23,1\end{array}$ & $\begin{array}{l}56,4 \\
78,9 \\
64,4 \\
47,4 \\
42,0 \\
57,6\end{array}$ & $\begin{array}{r}18,1 \\
63,3 \\
30,6 \\
6,4 \\
3,4 \\
4,1\end{array}$ & $\begin{array}{r}13,7 \\
8,8 \\
5,8 \\
4,4 \\
55,6 \\
6,1\end{array}$ & $\begin{array}{r}17,3 \\
21,4 \\
23,7 \\
8,3 \\
16,5 \\
17,2\end{array}$ \\
\hline $\begin{array}{l}\text { Groupe V - Semaine standard } \\
\text { fréquente } \\
45 \text { Prof. interm. adm. fonction publique } \\
47 \text { Techniciens } \\
48 \text { Contremaîtres } \\
52 \mathrm{~A} \text { Employés adm. Fonction publique } \\
\text { 52B Agents de service Fonction } \\
\text { publique } \\
54 \text { Employés adm. des entreprises } \\
63 \text { Ouvriers qualifiés de type artisanal } \\
68 \text { Ouvriers non qual. de type artisanal } \\
69 \text { Ouvriers agricoles }\end{array}$ & $\begin{array}{l}34,2 \\
32,9 \\
35,8 \\
40,3 \\
32,5 \\
47,1 \\
27,8 \\
34,6 \\
32,3 \\
41,9\end{array}$ & $\begin{array}{r}16,6 \\
21,4 \\
17,7 \\
18,2 \\
15,2 \\
\\
10,3 \\
20,1 \\
15,0 \\
6,7 \\
16,3\end{array}$ & $\begin{array}{l}30,5 \\
36,1 \\
34,4 \\
35,2 \\
24,4 \\
\\
24,9 \\
34,6 \\
29,9 \\
15,5 \\
25,6\end{array}$ & $\begin{array}{l}70,9 \\
76,6 \\
84,3 \\
81,8 \\
63,6 \\
67,7 \\
71,4 \\
62,5 \\
58,7 \\
74,4\end{array}$ & $\begin{array}{r}26,6 \\
24,7 \\
38,1 \\
42,1 \\
20,3 \\
6,6 \\
26,5 \\
20,2 \\
20,2 \\
41,9\end{array}$ & $\begin{array}{r}37,7 \\
23,8 \\
46,8 \\
51,6 \\
29,2 \\
\\
13,0 \\
29,2 \\
51,1 \\
36,2 \\
33,7\end{array}$ & $\begin{array}{l}52,2 \\
66,2 \\
67,0 \\
63,5 \\
48,0 \\
40,6 \\
55,4 \\
34,9 \\
42,7 \\
64,4\end{array}$ & $\begin{array}{r}13,9 \\
37,0 \\
24,1 \\
28,9 \\
10,0 \\
\\
5,2 \\
12,8 \\
3,8 \\
1,3 \\
2,0\end{array}$ & $\begin{array}{l}2,2 \\
0,0 \\
3,4 \\
3,2 \\
1,7 \\
\\
4,7 \\
1,0 \\
2,3 \\
3,1 \\
0,4\end{array}$ & $\begin{array}{r}9,3 \\
11,6 \\
12,2 \\
11,9 \\
11,4 \\
4,8 \\
6,2 \\
7,1 \\
7,8 \\
16,9\end{array}$ \\
\hline $\begin{array}{l}\text { Groupe VI - Horaires souvent décalés } \\
53 \text { Policiers, militaires } \\
62 \text { Ouvriers qualifiés de type industriel } \\
64 \text { C Chauffeurs sauf poids lourds } \\
64 \text { L Chauffeurs de poids lourds } \\
65 \text { Ouvr. qual. de la manut., } \\
\text { du magas. et du transp. } \\
67 \text { Ouvriers non qual. de type industriel }\end{array}$ & $\begin{array}{l}38,3 \\
63,8 \\
32,0 \\
39,6 \\
61,9 \\
\\
31,8 \\
36,3\end{array}$ & $\begin{array}{r}12,0 \\
9,6 \\
13,3 \\
5,7 \\
12,1 \\
\\
12,1 \\
12,3\end{array}$ & $\begin{array}{l}\mathbf{1 7 , 2} \\
13,3 \\
19,1 \\
18,9 \\
22,0 \\
18,3 \\
13,8\end{array}$ & $\begin{array}{l}54,9 \\
60,8 \\
59,1 \\
62,3 \\
55,9 \\
\\
54,2 \\
45,8\end{array}$ & $\begin{array}{l}16,8 \\
13,7 \\
20,7 \\
17,0 \\
10,0 \\
13,1 \\
14,8\end{array}$ & $\begin{array}{l}53,0 \\
18,0 \\
60,8 \\
47,2 \\
61,8 \\
53,3 \\
51,2\end{array}$ & $\begin{array}{l}32,5 \\
67,1 \\
30,7 \\
56,6 \\
42,0 \\
\\
28,0 \\
19,3\end{array}$ & $\begin{array}{r}4,1 \\
16,6 \\
3,8 \\
1,9 \\
1,7 \\
\\
4,7 \\
1,6\end{array}$ & $\begin{array}{l}\mathbf{1 , 8} \\
4,0 \\
1,6 \\
1,2 \\
1,7 \\
\\
0,5 \\
1,9\end{array}$ & $\begin{array}{r}9,9 \\
24,0 \\
8,1 \\
16,0 \\
19,2\end{array}$ \\
\hline
\end{tabular}

Lecture : pour la signification des intitulés de colonne, se reporter au tableau 3. Ainsi, les professeurs effectuent 45,2 \% de leur travail a domicile, et $98 \%$ d'entre eux rapportent du travail à domicile. La variation des horaires hebdomadaires d'une semaine sur l'autre est maximale pour les agriculteurs (fourchette de $29,7 \%$ ).

Champ : actifs occupés à temps plein et décrivant une semaine normale de travail.

Source : enquête Emploi du temps, 1998-1999, Insee. 
Le sentiment d'être fatigué du fait de la lourdeur des horaires de travail est plus rarement exprimé par les personnes effectuant un horaire standard (28\%) que par les autres, même lorsque cellesci bénéficient d'une semaine de quatre jours pourtant nettement plus brève en moyenne (33,7 h) qu'une semaine standard (40,2 h) (cf. tableau 7). Le sentiment de manquer de temps, que ce soit au travail ou dans la vie quotidienne, touche moins souvent les personnes à horaires standard que celles à semaines de quatre jours. Les personnes effectuant des horaires irréguliers ont dans l'ensemble des semaines de travail plus lourdes que celles à horaires réguliers (Fermanian et Baesa, 1997). Pour caractériser l'effet propre du type d'horaire, régulier ou non, il est nécessaire de neutraliser l'effet de la durée du travail.

\section{Être débordé résulte à la fois des charges professionnelles et domestiques}

Des régressions multiples permettent de prendre en compte simultanément les effets de la durée $\mathrm{du}$ travail et ceux du type d'organisation des horaires sur la fatigue perçue, ainsi que sur le

\section{Graphique V \\ Fatigue et manque de temps selon la charge de travail}

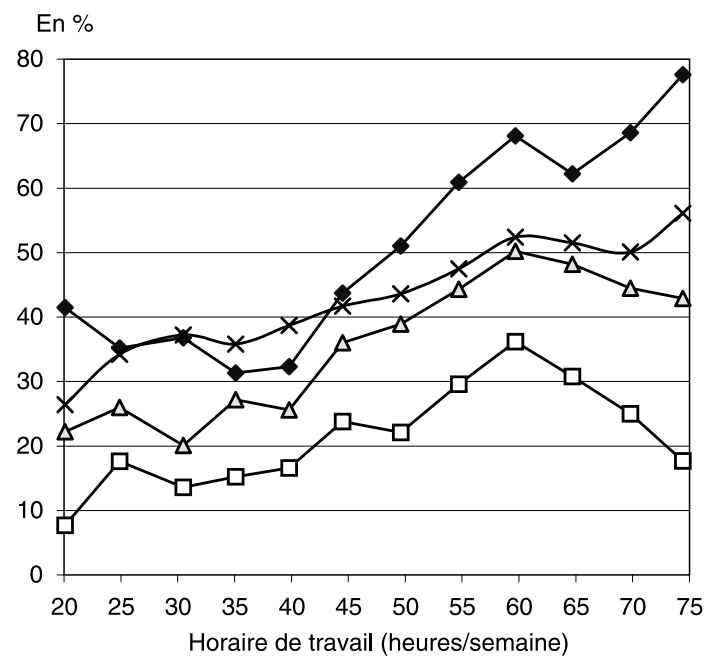

— Fatigué à cause de la durée quotidienne de travail - $\square$ - Débordé

$\neg-$ Manque de temps au travail

$\leftarrow$ Manque de temps dans la vie quotidienne

Lecture : $30 \%$ des actifs travaillant 55 heures par semaine se sentent débordés dans leur travail.

Champ : actifs occupés à temps plein et décrivant une semaine normale de travail.

Source : enquête Emploi du temps, 1998-1999, Insee. sentiment d'être débordé ou de manquer de temps. Dans cette analyse, on se limite à la population travaillant un nombre d'heures proche de la moyenne générale, conformément à l'un des critères de définition de la semaine standard, de $35 \mathrm{~h}$ à moins de $45 \mathrm{~h}(49,4 \%$ des actifs à temps plein se situent dans cet intervalle).

La durée du travail étant contrôlée, le sentiment d'être fatigué en fin de journée du fait de la lourdeur des horaires de travail est significativement plus rare chez les personnes dont les horaires sont standard que chez les autres ; il est aussi plus rare lorsque les horaires de travail varient peu, pour une même personne d'une semaine à une autre - c'est-à-dire lorsque l'indicateur correspondant est peu élevé (cf. tableau 8). La présence d'enfants au foyer va de pair avec une plus grande fréquence du sentiment de fatigue, et, chez les femmes en particulier, de celui d'être débordé, de manquer de temps dans la vie quotidienne. Les femmes expriment davantage leur sentiment de fatigue liée à la longueur des horaires de travail, et cette différence est très significative. Le sentiment d'être débordé résulte en effet à la fois des charges de travail professionnelles et domestiques.

\section{L'autonomie s'accompagne d'une plus grande difficulté à gérer son temps sans se sentir débordé}

Rapporter du travail à faire à la maison accroît significativement la fréquence du sentiment d'être débordé ou de manquer de temps. L'association est nette entre le niveau de formation (position sur une échelle à quatre niveaux, sans diplôme ou CEP, CAP ou BEP, baccalauréat, diplôme supérieur au bac) et le sentiment de manquer de temps, que ce soit au travail ou dans la vie quotidienne. Cet effet significatif se maintient lorsqu'on introduit parmi les régresseurs des indicateurs d'autonomie dans la disposition du temps (liberté de s'absenter pendant plusieurs heures durant le travail, liberté d'interrompre le travail en dehors des pauses instituées). Ces indicateurs d'autonomie n'ont d'effet significatif que lorsque le niveau de formation est retiré de la liste des variables explicatives (7). Les personnes qui organisent leur temps de manière autonome déclarent plus souvent manquer de temps ou être débordées. Mais

7. Ces analyses non reproduites ici sont disponibles auprès de l'auteur. 
Tableau 7

Fatigue et manque de temps selon le type d'horaire

\begin{tabular}{|l|c|c|c|c|c|c|}
\hline & Effectif & $\begin{array}{c}\text { Durée } \\
\text { hebdomadaire } \\
\text { du travail } \\
(1)\end{array}$ & $\begin{array}{c}\text { Fatigué à } \\
\text { cause durée } \\
\text { du travail } \\
(2)\end{array}$ & $\begin{array}{c}\text { Débordé } \\
\text { (2) }\end{array}$ & $\begin{array}{c}\text { Manque } \\
\text { de temps } \\
\text { au travail } \\
\text { (2) }\end{array}$ & $\begin{array}{c}\text { Manque } \\
\text { de temps } \\
\text { dans la vie } \\
\text { quotidienne (2) }\end{array}$ \\
\hline Semaine de 6 ou 7 jours de travail, sauf nuit & 1054 & 53,7 & 52,7 & 26,8 & 39,2 & 45,1 \\
Semaine avec travail de nuit & 239 & 48,2 & 51,1 & 13,3 & 23,6 & 33,5 \\
Semaine de 5 jours, ni standard ni nuit & 1003 & 44,5 & 52,0 & 24,0 & 37,8 & 43,7 \\
Semaine standard & 1350 & 40,2 & 28,4 & 16,2 & 25,4 & 37,6 \\
Semaine de 4 jours, sauf nuit & 449 & 33,7 & 37,7 & 15,3 & 32,6 & 41,1 \\
Autres semaines & 129 & 21,0 & 40,4 & 18,8 & 27,6 & 38,2 \\
\hline Ensemble & $\mathbf{4 2 2 4}$ & $\mathbf{4 3 , 8}$ & $\mathbf{4 2 , 7}$ & $\mathbf{2 0 , 5}$ & $\mathbf{3 2 , 5}$ & $\mathbf{4 1 , 1}$ \\
\hline 1. Nombre d'heures par semaine figurant au semainier. & & & & \\
2. En \%.
\end{tabular}

Lecture : pour la définition des types de semaine, on se reportera à l'encadré 2. Pour les actifs occupés à temps plein, la durée moyenne d'une semaine normale standard est de 40,2 h. 28,4\% de ces personnes se déclarent fatiguées à cause de la durée du travail.

Champ : actifs occupés à temps plein et décrivant une semaine normale de travail.

Source : enquête Emploi du temps, 1998-1999, Insee.

Tableau 8

Fatigue et manque de temps selon la charge de travail, le type d'horaire et les caractéristiques individuelles

\begin{tabular}{|c|c|c|c|c|c|c|c|c|c|c|c|c|}
\hline \multirow[b]{2}{*}{$\begin{array}{l}\text { Variables } \\
\text { explicatives }\end{array}$} & \multicolumn{3}{|c|}{$\begin{array}{c}\text { Se sentir fatigué en fin de } \\
\text { journée à cause de la durée } \\
\text { de la journée de travail }\end{array}$} & \multicolumn{3}{|c|}{ Se sentir débordé } & \multicolumn{3}{|c|}{$\begin{array}{l}\text { Manquer souvent de } \\
\text { temps au travail }\end{array}$} & \multicolumn{3}{|c|}{$\begin{array}{c}\text { Manquer souvent de } \\
\text { temps dans la vie } \\
\text { quotidienne }\end{array}$} \\
\hline & $\begin{array}{c}\text { Hom- } \\
\text { mes }\end{array}$ & $\begin{array}{c}\text { Fem- } \\
\text { mes }\end{array}$ & $\begin{array}{c}\text { Ensem- } \\
\text { ble }\end{array}$ & $\begin{array}{l}\text { Hom- } \\
\text { mes }\end{array}$ & $\begin{array}{l}\text { Fem- } \\
\text { mes }\end{array}$ & $\begin{array}{c}\text { Ensem- } \\
\text { ble }\end{array}$ & $\begin{array}{l}\text { Hom- } \\
\text { mes }\end{array}$ & $\begin{array}{c}\text { Fem- } \\
\text { mes }\end{array}$ & $\begin{array}{c}\text { Ensem- } \\
\text { ble }\end{array}$ & $\begin{array}{l}\text { Hom- } \\
\text { mes }\end{array}$ & $\begin{array}{l}\text { Fem- } \\
\text { mes }\end{array}$ & $\begin{array}{c}\text { Ensem- } \\
\text { ble }\end{array}$ \\
\hline $\begin{array}{l}\text { Durée du travail } \\
\text { professionnel }\end{array}$ & ++ & +++ & +++ & + & + & ++ & + & + & ++ & ns & ns & ns \\
\hline $\begin{array}{l}\text { Rapporter du travail } \\
\text { à la maison }\end{array}$ & ns & ns & ns & ++ & +++ & +++ & ++ & +++ & +++ & + & + & ++ \\
\hline Niveau de formation & ns & ns & ns & ns & + & + & ++ & + & +++ & +++ & +++ & +++ \\
\hline $\begin{array}{l}\text { Nombre d'enfants } \\
\text { âgés de moins de } \\
18 \text { ans }\end{array}$ & + & + & + & + & ++ & ++ & + & ns & + & ++ & +++ & +++ \\
\hline Semaine standard & $-\cdots$ & $-\cdots$ & $-\cdots$ & ns & ns & - & - & ns & -- & ns & ns & ns \\
\hline Fourchette & +++ & +++ & +++ & ns & ns & ns & ns & ns & + & ns & ns & ns \\
\hline Sexe féminin & $/ /$ & $/ /$ & +++ & $/ /$ & $/ /$ & +++ & $/ /$ & $/ /$ & ++ & $/ /$ & $/ /$ & +++ \\
\hline
\end{tabular}

Lecture : +++, - - - : effet significatif (positif ou négatif) au seuil de 0,001. ++, - - : ... au seuil de 0,01. +, - : ... au seuil de 0,1. ns : non significatif au seuil de 0,1 . Ces effets sont mis en évidence au moyen de régressions logistiques. Ainsi, toute choses égales par ailleurs, une forte variabilité des horaires d'une semaine sur l'autre (se traduisant par une valeur élevée de l'indicateur correspondant (fourchette)) va de pair avec une plus grande fréquence du sentiment de fatigue en fin de journée du fait de la durée de la journée de travail.

Champ : 1254 hommes et 870 femmes travaillant à temps plein, ayant fourni un semainier couvrant une semaine normale de travail faisant apparaître un horaire au moins égal à 35 h et inférieur à $45 h$.

Source : enquête Emploi du temps, 1998-1999, Insee.

ce lien n'existe que parce que ces personnes sont plus diplômées que les autres : un niveau de formation élevé va de pair à la fois avec plus d'autonomie dans l'organisation du temps de travail et avec de plus grandes difficultés à gérer le temps sans se sentir débordé. Cette configuration s'observe à durée du travail contrôlée - par ailleurs une plus grande liberté dans la détermi- nation des horaires va de pair avec des durées du travail en moyenne plus élevées (Fermanian, 1999).

En revanche, l'âge, le statut (salarié de l'État, de grands ou petits établissements, non-salarié) n'ont pas ou ont peu d'effets nets sur le sentiment de manque de temps. 


\section{BIBLIOGRAPHIE}

Boisard P. et Fermanian J.-D. (1999), « Publicprivé : les écarts des durées du travail réellement pratiquées », Centre d'Études de l'Emploi, n 36.

Bonnet G. et Murcia S. (1996), «Temps et charge de travail estimés par les enseignants du second degré dans les établissements publics », Éducation et formations, $\mathrm{n}^{\circ} 46$.

Bue J. et Rougerie C. (1999), «L'organisation des horaires : un état des lieux en mars 1998 », Premières informations et premières synthèses, $\mathrm{n}^{\circ}$ 99.07, Dares.

Dumontier F. et Pan Ke Shon J.-L. (1999), « En 13 ans, moins de temps contraint et plus de loisirs », Insee Première, n ${ }^{\circ} 675$.

Dumontier F. et Pan Ke Shon J.-L. (2000), «Enquête Emploi du temps 1998-1999. Descrip- tion des activités quotidiennes », Insee Résultats, $n^{\circ}$ 693-694, Insee.

Fermanian J.-D. (1999), « Le temps de travail des cadres », Insee Première, $n^{\circ} 671$.

Fermanian J.-D., Baesa M.-P. (1997), « La durée $\mathrm{du}$ travail à temps complet », Insee Première, $n^{\circ} 545$.

Insee (1992), Nomenclature des professions et catégories socioprofessionnelles, Paris, 2 tomes.

Guillaume F.-R. (2000), « Conditions de vie et de travail des enseignants », Éducation et formations, $\mathrm{n}^{\circ} 56$.

Missègue N. (2000), «Le temps de travail des indépendants », Insee Première, n ${ }^{\circ} 695$. 
